\title{
Partitioning dysprosium's electronic spin to reveal entanglement in nonclassical states
}

\author{
Tanish Satoor $\oplus^{*}$, Aurélien Fabre, ${ }^{*}$ Jean-Baptiste Bouhiron ${ }^{\circledR}$, Alexandre Evrard, Raphael Lopes ${ }^{\circledR}$, and Sylvain Nascimbene ${ }^{\dagger}$ \\ Laboratoire Kastler Brossel, Collège de France, CNRS, ENS-PSL University, Sorbonne Université, \\ 11 Place Marcelin Berthelot, 75005 Paris, France
}

(Received 30 April 2021; revised 9 July 2021; accepted 20 August 2021; published 1 October 2021)

\begin{abstract}
Quantum spins of mesoscopic size are a well-studied playground for engineering nonclassical states. If the spin represents the collective state of an ensemble of qubits, its nonclassical behavior is linked to entanglement between the qubits. In this paper, we report on an experimental study of entanglement between two subsystems of dysprosium's electronic spin. Its ground state, of angular momentum $J=8$, can formally be viewed as a set of $2 J$ qubits symmetric upon exchange. To access entanglement properties, we partition the spin by optically coupling it to an excited state $J^{\prime}=J-1$, which removes a pair of qubits in a state defined by the light polarization. Starting with the well-known $\mathrm{W}$ and squeezed states, we extract the concurrence of qubit pairs, which quantifies their nonclassical character. We also directly demonstrate entanglement between the 14- and 2-qubit subsystems via an increase in entropy upon partition. In a complementary set of experiments, we probe decoherence of a state prepared in the excited level $J^{\prime}=J+1$ and interpret spontaneous emission as a loss of a qubit pair in a random state. This allows us to contrast the robustness of nonclassical pairwise correlations of the W state with the fragility of the coherence involved in a Schrödinger cat state. Our findings open up the possibility to engineer novel types of entangled atomic ensembles, in which entanglement occurs within each atom's electronic spin as well as between different atoms. Qubit ensembles with large entanglement depth could then be realized with a few atoms only, facilitating the scaling up of quantum-enhanced sensors.
\end{abstract}

DOI: 10.1103/PhysRevResearch.3.043001

\section{INTRODUCTION}

Entanglement is a hallmark of nonclassical behavior in compound quantum systems. Minimal entangled systems of qubit pairs, as realized with correlated photon pairs, play a central role in testing the foundations of quantum mechanics $[1,2]$. Entanglement can also be engineered in many-particle systems [3], such as an ensemble of interacting atoms [4]. In this case, the atoms are not individually addressable, and quantum correlations are indirectly revealed by measuring global properties, such as a squeezed spin projection quadrature [5-8] or via the quantum enhancement of magnetic sensitivity [9-11]. State-of-the-art experiments on photonic systems [12], superconducting qubits [13], trapped ions [14], and Rydberg atom arrays [15] can now produce highly entangled states of tens of individually identifiable qubits, in which entanglement is more readily observable.

Besides quantum state tomography, a wide array of methods have been developed for the detection of entanglement $[16,17]$. In two-qubit systems, the degree of entanglement is quantified by the concurrence $[18,19]$. Its direct measurement remains challenging since it requires nonlinear operations on

\footnotetext{
*These authors contributed equally to this work.

†sylvain.nascimbene@lkb.ens.fr
}

Published by the American Physical Society under the terms of the Creative Commons Attribution 4.0 International license. Further distribution of this work must maintain attribution to the author(s) and the published article's title, journal citation, and DOI. the prepared state [20-23], and it was so far only achieved for photon pairs in pure quantum states [21]. In the case of multipartite systems, the study of entanglement is cumbersome due to the existence of distinct classes of entanglement [24]. It is often revealed using entanglement witnesses, by measuring the fidelity with respect to a given entangled state [25] - the method being limited to simple enough target states.

In this paper, we study quantum entanglement between subsystems of the electronic spin of dysprosium atoms, of angular momentum $J=8$ in its ground state and prepared in nonclassical spin states. Quantum states with nonclassical correlations have been extensively studied in single large-spin systems, including photon qutrits [26], ground-state atomic spins [27,28], molecules [29], and Rydberg atoms [30]. In the formal analogy between a spin $J$ and a set of $2 J$ qubits symmetric upon exchange [31], nonclassicality goes hand in hand with entanglement between the virtual qubits. However, as long as the angular momentum $J$ is conserved, the qubit ensemble cannot be partitioned, and the relevance of entanglement is disputable. Here, we use an optical coupling to an excited electronic state of angular momentum $J^{\prime}=J-1$ to partition the 16-qubit ensemble associated with the spin $J$, giving access to entanglement. The virtual absorption of a photon is interpreted as the annihilation of a qubit pair in a state defined by the light polarization, leaving a set of 14 qubits in the excited electronic level [see Fig. 1(a)]. This process thus realizes a partition of the electronic spin $J$ in two subsystems - the excited electronic spin $J^{\prime}=J-1$ and the photon angular momentum $L=1$. We use this partition to probe entanglement in nonclassical spin states, either by characterizing nonclassical behavior of qubit pairs via the 


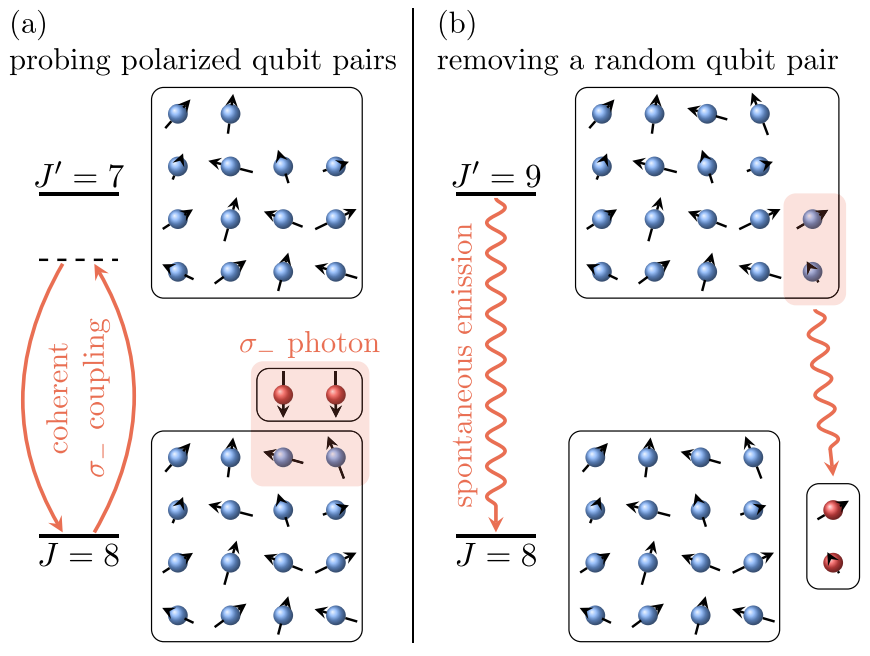

FIG. 1. Scheme of the experiments manipulating qubit pairs in the electronic spin of dysprosium. An electronic spin of angular momentum $J$ can be viewed as a set of $2 J$ virtual qubits symmetric upon exchange. (a) The coherent coupling to an excited state $J^{\prime}=J-1$ with $\sigma_{-}$polarized light probes the probability to find a qubit pair polarized in $|\uparrow \uparrow\rangle_{z}$. (b) The spontaneous emission from an excited state $J^{\prime}=J+1$ removes a random pair of qubits.

measurement of concurrence or by revealing an increase in entropy upon partition. We extend this protocol to probe decoherence in states prepared in an excited electronic level $J^{\prime}=J+1$ [see Fig. 1(b)]. There, the spontaneous emission of a photon drives the system to the electronic ground state $J$, which corresponds to the removal of a qubit pair randomly drawn from the initial state. We reveal the robustness of nonclassical pairwise correlations with respect to qubit loss, as well as the fragility of coherence in Schrödinger cat states.

This paper is organized as follows. We present in Sec. II the experimental protocol used to measure the properties of qubit pairs extracted from the electronic spin, based on the polarization dependence of the light-spin interaction. In Sec. III, we investigate the nonclassical character of these qubit pairs via the measurement of the concurrence of the reduced two-qubit density matrix and apply it to a $\mathrm{W}$ state and a squeezed state. In Sec. IV, we investigate the increase of entropy upon the 14|2 partition as a proof of entanglement for $\mathrm{W}$ and Schrödinger cat states, by studying the mixed nature of the reduced two-qubit density matrix. In Sec. V, we study the decoherence upon the loss of a qubit pair triggered by spontaneous emission. We show that nonclassical pairwise correlations are robust with respect to the extraction of qubits. In contrast, the coherence of a Schrödinger cat state is completely destroyed upon qubit loss, due to the complete which path information carried by the spontaneously emitted photon's polarization. In another superposition state, we show the existence of a quantum jump leaving the path information hidden, such that maximal-order coherence remains visible. Finally, we present a possible extension of our work to ensembles of dysprosium atoms entangled together using an optical resonator. Such systems would combine entanglement between atoms and within each electronic spin, allowing one to scale up entanglement depth and its application to quantumenhanced sensing.

\section{PAIR HUSIMI FUNCTION MEASUREMENT}

\section{A. Probing pairs via light coupling}

The electronic ground state $J=8$ can be interpreted as the sum of $2 J=16$ virtual spin- $1 / 2 \mathrm{~s}$, in a state symmetric upon exchange. We discuss here the partition of this qubit ensemble, prepared in a state $\rho$, through the coupling to an excited electronic level, of angular momentum $J^{\prime}=7$. As sketched in Fig. 1(a), the coupling to the excited manifold is induced by light close to the optical transition, via the absorption of a photon. The photon polarization $\epsilon$ defines an $L=1$ quantum state $|\boldsymbol{\epsilon}\rangle$ that can be considered as a symmetric two-qubit state. We restrict ourselves here to the case of a circular polarization $\sigma_{-}$, which corresponds to qubits polarized in $|\downarrow \downarrow\rangle_{z}$. Since the excited state contains only $2 J^{\prime}=14$ qubits, two qubits are removed upon photon absorption. The conservation of angular momentum requires these removed qubits to be polarized in $|\uparrow \uparrow\rangle_{z}$, the time-reversed state of the absorbed photon's polarization. The excited state $\rho^{\prime}$ can be then written as a projected state $\rho^{\prime}=\left\langle\left.\uparrow \uparrow\right|_{z} \rho \mid \uparrow \uparrow\right\rangle_{z}$. The probability for a pair chosen from the 16 qubits to be polarized in $|\uparrow \uparrow\rangle_{z}$ then reads

$$
Q_{\text {pair }}\left(\mathbf{e}_{z}\right)=\operatorname{Tr} \rho^{\prime},
$$

defining the pair Husimi function along the direction $\mathbf{e}_{z}$. Hence the light absorption properties of the electronic spin $J$ can be linked to the properties of its two-qubit reduced density matrix.

To probe this behavior, we measure the light shift $V$ induced by an off-resonant light beam close to the considered optical transition. The light shift, being induced by virtual photon absorption processes, is proportional to the pair Husimi function, as

$$
V / V_{0}=Q_{\text {pair }}\left(\mathbf{e}_{z}\right), \quad V_{0}=\frac{(d E)^{2}}{\hbar \Delta},
$$

where $d=\langle J-1|| \mathbf{d} \| J\rangle$ is the reduced dipole matrix element, $E$ is the light electric field amplitude, and $\Delta$ is the detuning from resonance.

\section{B. Application to Dicke states}

We illustrate our method by measuring the value of the Husimi function $Q_{\text {pair }}\left(\mathbf{e}_{z}\right)$ for an arbitrary Dicke state $|m\rangle$ (with $-J \leqslant m \leqslant J$ ), which we denote $Q_{m}$ hereafter.

All our experiments are performed on a cloud of $1.0(1) \times$ $10^{5}$ dysprosium atoms (of the bosonic isotope ${ }^{162} \mathrm{Dy}$ ), held in an optical dipole trap at a temperature $T=0.54(3) \mu \mathrm{K}$. The results described in this paper can be understood by considering a single atom, with the ensemble acting as an averaging mechanism only. The experimental scheme for the $Q_{m}$ measurement is shown in Fig. 2(a). We prepare the atoms in a coherent state $|m=J\rangle_{\mathbf{n}}$ polarized along a direction $\mathbf{n}$, parametrized by the spherical angles $(\theta, \phi)$. The polar angle $\theta$ determines the projection probabilities $\Pi_{m}$ along the Dicke states $|m\rangle$, which are significant for values of $m$ close to $J \cos \theta$. We then push the atomic cloud by applying an off-centered laser beam, with circular $\sigma_{-}$polarization and blue detuning with respect to an optical transition at $696 \mathrm{~nm}$. The intensity gradient then leads to a force along $x$ proportional to the light shift [Fig. 2(a)]. After this kick, a magnetic 

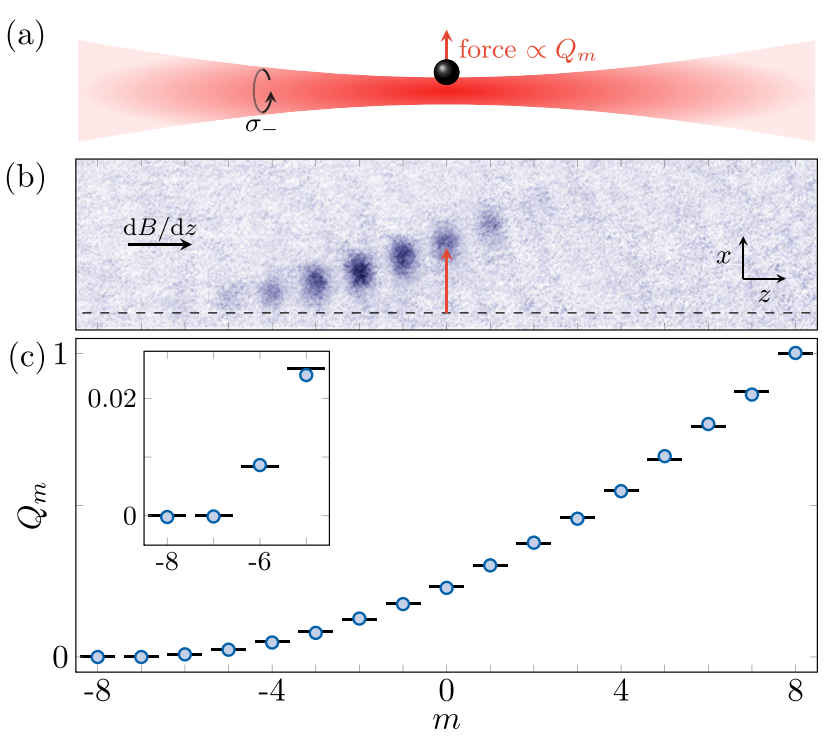

FIG. 2. Husimi function measurement for Dicke states. (a) Scheme of the light shift measurement. We measure the force induced on the atoms by an off-centered laser beam, blue detuned with respect to the optical resonance. (b) Image of an atomic gas prepared in a coherent state of polar angle $\theta \simeq 100^{\circ}$. The atoms are kicked along $x$ by the laser beam. Subsequently, we apply a magnetic field gradient separating the magnetic sublevels $|m\rangle$ along $z$ during time of flight. The dashed line indicates the mean $x$ position in the absence of the repulsive laser beam. (c) Probability $Q_{m}$ for a qubit pair taken in the Dicke state $|m\rangle$ to be in $|\uparrow \uparrow\rangle_{z}$, deduced from the kick amplitudes. In all figures, error bars represent the $1 \sigma$ statistical uncertainty (here smaller than the blue circles). The black lines are the theoretical values of Eq. (1).

field gradient is applied to spatially separate the different $m$ components along $z$, which allows us to retrieve the light shift experienced by each Dicke state independently. After a 2.3-ms time of flight, we image the atoms and measure the $x$ displacement for each Dicke state $|m\rangle$ that is significantly populated, and hence their values $Q_{m}$. A typical absorption image is shown in Fig. 2(b). Repeating this measurement for various angles $\theta$, we measure the light shifts for all projections $m$ and infer the $Q_{m}$ values shown in Fig. 2(c) [32].

Our measurements are consistent with an absence of light shift for the states $|m=-J\rangle$ and $|m=-J+1\rangle$; that is, these states are dark with respect to the $J \rightarrow J^{\prime}=J-1$ optical transition for $\sigma_{-}$polarized light. In terms of the underlying qubits, the state $|m=-J\rangle$ only contains $|\downarrow\rangle_{z}$-polarized qubits, while the state $|m=-J+1\rangle$ has a single qubit in $|\uparrow\rangle_{z}$. In both cases, a qubit pair cannot be found polarized in $|\uparrow \uparrow\rangle_{z}$; hence $Q_{-J}=Q_{-J+1}=0$.

More generally, a Dicke state $|m\rangle$ is composed of $J-m$ qubits in $|\downarrow\rangle_{z}$ and $J+m$ qubits in $|\uparrow\rangle_{z}$ [33]. The probability to pick a pair $|\uparrow \uparrow\rangle_{z}$ simply reads

$$
Q_{m}=\left(\begin{array}{c}
J+m \\
2
\end{array}\right) /\left(\begin{array}{c}
2 J \\
2
\end{array}\right)=\frac{(J+m)(J+m-1)}{2 J(2 J-1)},
$$

in good agreement with our measurements.

We use these measurements to probe the Husimi function of states lacking $z$ rotation symmetry. For this, we measure their projection probabilities $\Pi_{m}(\mathbf{n})$ along $\mathbf{n}$ by combining a spin rotation and a Stern-Gerlach projective measurement along $z$. We then infer the Husimi function by weighting these probabilities with the $Q_{m}$ values, as

$$
Q_{\text {pair }}(\mathbf{n})=\sum_{m} Q_{m} \Pi_{m}(\mathbf{n}) .
$$

In the following, we use the theoretical values of Eq. (1) rather than the measured ones to avoid propagating systematic errors.

\section{Coherent and W states}

We first apply the above protocol to the quasiclassical coherent spin state $|m=-J\rangle$ and the $\mathrm{W}$ state $|m=-J+1\rangle$. The coherent state can be viewed as a set of $2 J$ qubits polarized in $|\downarrow\rangle_{z}$, forming a nonentangled product state. The $\mathrm{W}$ state, which hosts a single qubit in $|\uparrow\rangle_{z}$, is a paradigmatic state of a fundamental class of entanglement [24], which has been realized and studied in various settings [34-41].

In our experiment, the atoms are initially spin polarized in the coherent state $|m=-J\rangle$. To produce the W state, we confine the system to the two spin states $|m=-J\rangle$ and $|m=-J+1\rangle$ by applying a strong quadratic light shift acting on the other spin states only, leading to a constrained quantum Zeno dynamics [42-44]. An additional resonant radiofrequency $\pi$ pulse then brings the system to $|m=-J+1\rangle$. The quadratic light shift is produced using the 696-nm laser beam with a $\sigma_{-}$polarization, leading to positive energy shifts for all Dicke states $|m\rangle$, except for $m=-J$ and $-J+1$. We reach a maximum W-state fidelity of $0.91(1)$, with residual overlaps on other Dicke states below 4\% [45].

We report in Figs. 3(a) and 3(b) the measured projection probabilities $\Pi_{m}(\theta)$ for these two states. For a given projection $m$, the coherent-state probabilities feature a single peak centered on the expected maximum at $\theta_{m}=\arccos (m / J)$, shown as red lines. For the $\mathrm{W}$-state probabilities, we observe a double-peaked distribution for all nonstretched states $m \neq \pm J$. This behavior results from the interference between two processes, depending on whether the spin $|\uparrow\rangle_{z}$ is projected on $|\uparrow\rangle_{\theta}$ or $|\downarrow\rangle_{\theta}$. The first (second) process dominates for $\theta \simeq 0$ $(\theta \simeq \pi)$, and the two processes destructively interfere at $\theta_{m}$, as observed in our data.

We combine these measurements to infer the pair Husimi functions using Eq. (2), finding good agreement with theory for both states [see Fig. 3(c)]. In particular, for the coherent state, our data match well the probability $Q_{\text {pair }}(\theta)=\sin ^{4}(\theta / 2)$ that two qubits in $|\downarrow\rangle_{z}$ are projected in $|\uparrow\rangle_{\theta}$. In the following sections we use these measurements to probe entanglement properties.

\section{NONCLASSICALITY OF QUBIT PAIRS}

Our first characterization of entanglement of the $2 J$-qubit state consists in revealing the nonclassical character of qubits pairs extracted from it.

\section{A. Measure of nonclassicality via the concurrence}

The collective state $\rho_{\text {pair }}$ of a qubit pair symmetric upon exchange can be written as the state of an angular momentum 

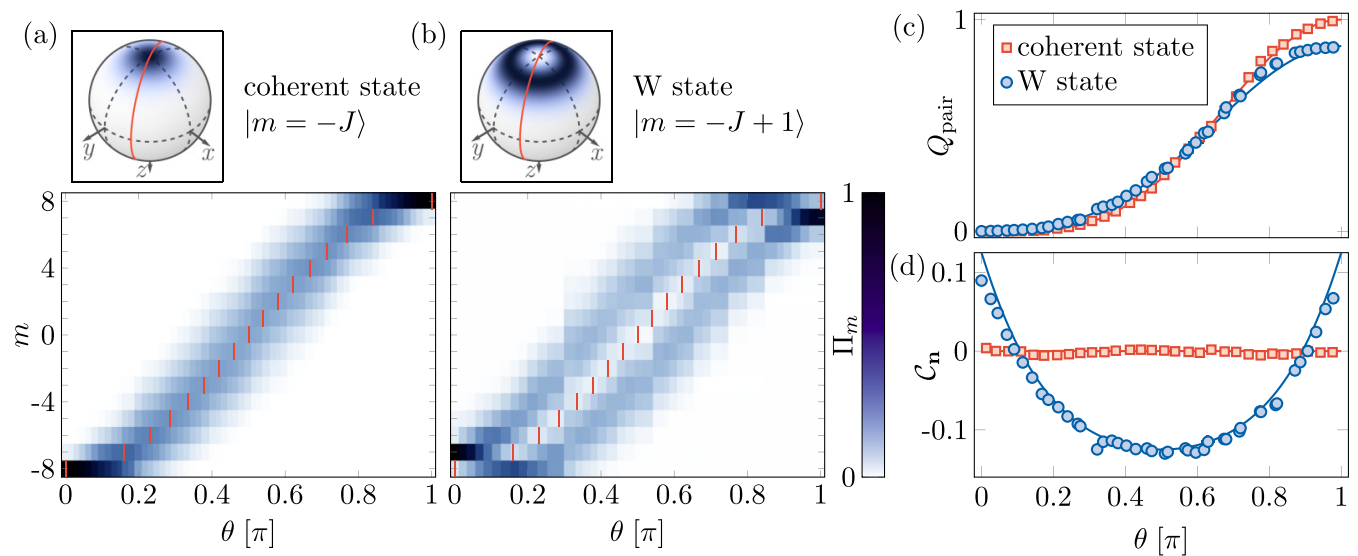

FIG. 3. Qubit pair properties of coherent and W states. (a) and (b) Measured spin projection probabilities $\Pi_{m}$ as a function of the polar angle $\theta$, for a coherent spin state (a) and for the $\mathrm{W}$ state (b). The red vertical lines indicate the expected maxima for the coherent state, also corresponding to minima for the $\mathrm{W}$ state. The top panels represent the considered spin- $J$ states on the Bloch sphere, where the red circles indicate the spanned measurement projection axis. (c) Pair Husimi function $Q_{\text {pair }}$ computed from the (a) and (b) data (blue circles and red squares, respectively). The lines correspond to the expected functions $Q_{\text {pair }}(\theta)$ for the coherent and $\mathrm{W}$ states (red and blue lines). (d) Distribution $\mathcal{C}_{\mathbf{n}}$ of nonclassical correlations as a function of the polar angle $\theta$. The points $\mathcal{C}_{\mathbf{n}}>0$ measured for the W state evidence nonclassicality.

$L=1$. Drawing an analogy with quantum optics [46,47], it will be called classical if it can be expressed as a statistical mixture of quasiclassical coherent states [48], as

$$
\left.\rho_{\text {pair }}^{\text {(classical) }}=\sum_{\mathbf{n}} w_{\mathbf{n}} \| \mathbf{n}\right\rangle\langle\mathbf{n} \|,
$$

where $\| \mathbf{n}\rangle$ is a spin-1 coherent state pointing along $\mathbf{n}$, and $w_{\mathbf{n}} \geqslant 0, \sum_{\mathbf{n}} w_{\mathbf{n}}=1$. Coherent states are the only pure states that satisfy the equality

$$
Z(\mathbf{n}) \equiv 2\left\langle L_{\mathbf{n}}^{2}\right\rangle-\left\langle L_{\mathbf{n}}\right\rangle^{2}-1=0
$$

for arbitrary measurement axis $\mathbf{n}$. Then it follows by convexity that $Z(\mathbf{n}) \geqslant 0$ for classical states. As shown in Ref. [48], the existence of a strictly negative value $Z(\mathbf{n})$ constitutes a necessary and sufficient criterion of nonclassicality.

To apply this criterion to our system, we use the connection between the mean values of spin projection and the Husimi function of qubit pairs extracted from the electronic spin $J$,

$$
\begin{aligned}
& \left\langle L_{\mathbf{n}}\right\rangle=Q_{\text {pair }}(\mathbf{n})-Q_{\text {pair }}(-\mathbf{n}), \\
& \left\langle L_{\mathbf{n}}^{2}\right\rangle=Q_{\text {pair }}(\mathbf{n})+Q_{\text {pair }}(-\mathbf{n}),
\end{aligned}
$$

leading to the expression $Z(\mathbf{n})=\alpha \mathcal{C}_{\mathbf{n}}$, where we introduce the coefficient $\alpha=\left(\sqrt{Q_{\text {pair }}(-\mathbf{n})}-\sqrt{Q_{\text {pair }}(\mathbf{n})}\right)^{2}-1$ and the distribution

$$
\mathcal{C}_{\mathbf{n}}=1-\left(\sqrt{Q_{\text {pair }}(-\mathbf{n})}+\sqrt{Q_{\text {pair }}(\mathbf{n})}\right)^{2} .
$$

The parameter $\alpha$ being negative, nonclassicality is characterized by the existence of a direction $\mathbf{n}$ for which $\mathcal{C}_{\mathbf{n}}$ is strictly positive. This criterion of nonclassicality is equivalent to the bipartite entanglement witness established in Ref. [49].

We show in Fig. 3(d) the distribution $\mathcal{C}_{\mathbf{n}}$ computed from the measured Husimi functions, for the coherent and $\mathrm{W}$ states. For these states, symmetric upon rotations around $z$, we expect $\mathcal{C}_{\mathbf{n}}$ to only depend on the polar angle $\theta$ of the measurement axis [50]. For the coherent state, the measured $\mathcal{C}_{\mathbf{n}}$ remains close to zero for all angles $\theta$. Indeed, qubit pairs drawn from this state form themselves a spin-1 coherent state, for which $\mathcal{C}_{\mathbf{n}}$ vanishes according to Eq. (4). For the $\mathrm{W}$ state, $\mathcal{C}_{\mathbf{n}}$ takes significantly positive values for $\theta$ close to 0 and $\pi$, showing a nonclassical character.

We now show that the distribution $\mathcal{C}_{\mathbf{n}}$ can be used to quantify the degree of nonclassicality of a quantum state, defined by its distance from the set of nonclassical states [51]. For a system of two qubits, this geometrical measure can be directly expressed in terms of the concurrence $\mathcal{C}$ [52], the most common measure of pairwise entanglement $[18,19]$. In our system, qubit pairs should be considered as indivisible quantum objects, such that the concurrence only measures the amount of nonclassical correlations. The concurrence can be explicitly written in terms of the density matrix, but it does not correspond to a directly accessible physical observable. Remarkably, the distribution $\mathcal{C}_{\mathbf{n}}$ can be used to retrieve the concurrence, as

$$
\mathcal{C}=\max \left[0, \max _{\mathbf{n}} \mathcal{C}_{\mathbf{n}}\right]
$$

This relation was conjectured and numerically checked for randomly generated states in Ref. [53].

For the $\mathrm{W}$ state realized in the experiment, the measured $\mathcal{C}_{\mathbf{n}}$ takes its maximum for $\theta=0$ leading to a concurrence $\mathcal{C}=$ $0.089(5)$. This value is about $71 \%$ of the maximum possible value $\mathcal{C}=1 / J=0.125$ in a system of $2 J$ qubits symmetric upon exchange [54], which would be reached for the $\mathrm{W}$ state in the absence of experimental imperfections. In our system, the concurrence is limited by the residual population $\Pi_{-J+2} \simeq$ 0.03 in the Dicke state $|m=-J+2\rangle$ that originates from spin-changing collisions between atoms in $|m=-J+1\rangle$.

\section{B. Pairwise correlations in a squeezed state}

Nonclassical correlations between qubit pairs play a central role in the squeezing of a spin projection quadrature [55]. In this section we extend the measurement of qubit pair properties to a squeezed spin state, which we produce via a nonlinear 

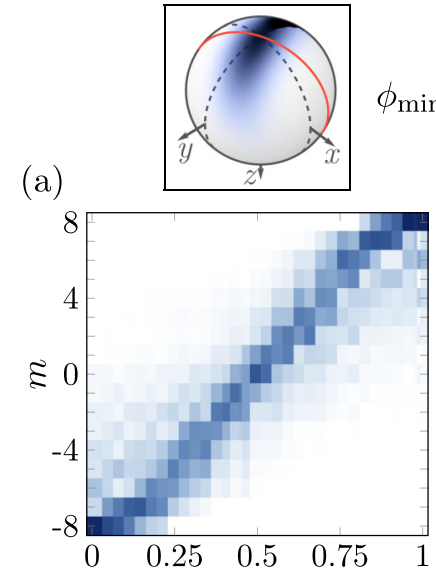

(b)
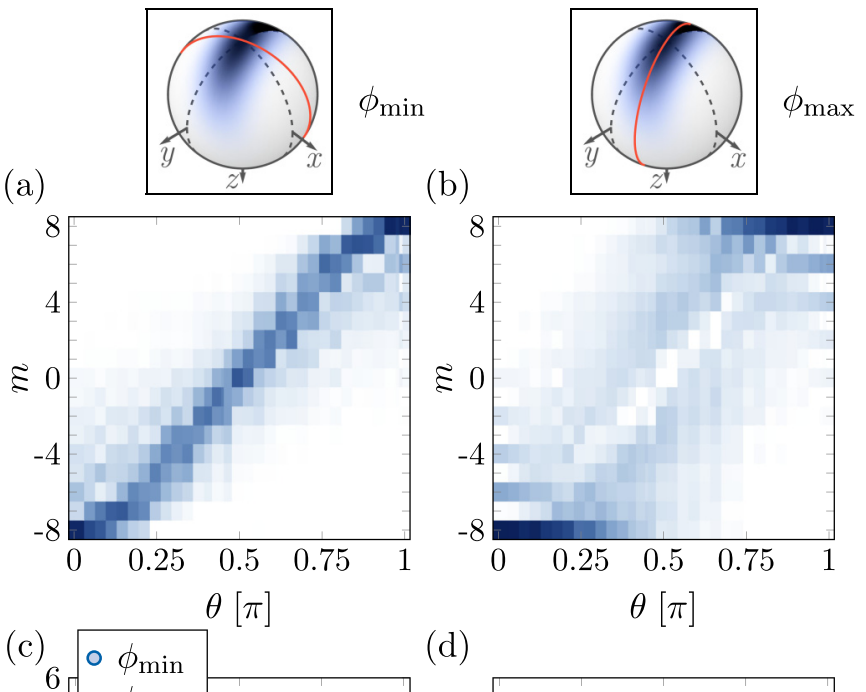

(d)

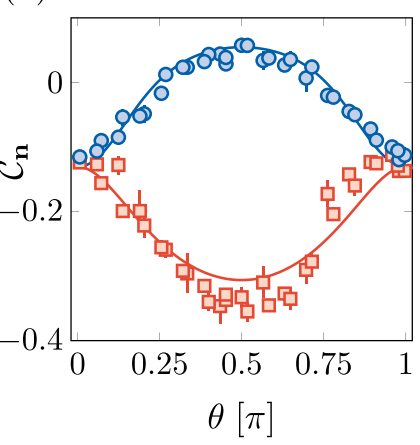

FIG. 4. Qubit pair properties for a squeezed state. (a) and (b) Measured spin projection probabilities $\Pi_{m}$ for a squeezed spin state, as a function of the polar angle $\theta$ with azimuthal angles $\phi_{\min }$ (a) and $\phi_{\max }$ (b). (c) Spin projection uncertainty $\Delta J_{\mathbf{n}}$ computed from the (a) and (b) data (blue circles and red squares, respectively). The lines correspond to the projection uncertainties expected for the targeted spin state. (d) Distribution $\mathcal{C}_{\mathbf{n}}$ of nonclassical correlations as a function of $\theta$.

spin dynamics. We apply a $\hbar \chi J_{x}^{2}$ spin coupling, generated by the spin-dependent light shift of the 696-nm laser beam, using a linear polarization $\mathbf{e}_{x}$ [56]. This coupling induces a twisting of the spin distribution, leading to the squeezing of a spin projection quadrature [55], as first implemented in atomic Bose-Einstein condensates [8,9]. In our experiment, we apply a nonlinear coupling of strength $\chi=2 \pi \times 32.1(4) \mathrm{kHz}$ for a duration $t \simeq 700 \mathrm{~ns}$, in the presence of a $z$ magnetic field $B=75(1) \mathrm{mG}$.

In contrast to the Dicke states discussed above, the spin projection probabilities are no longer invariant around $z$. We show in Figs. 4(a) and 4(b) the probabilities $\Pi_{m}(\theta, \phi)$ for two azimuthal angles $\phi_{\min }=-0.4(2) \mathrm{rad}$ and $\phi_{\max }=\phi_{\min }+$ $\pi / 2$, which feature minimal and maximal spin projection uncertainties, respectively. For $\theta=\pi / 2$, a minimum spin projection uncertainty $\Delta J_{\min }=0.92(16)$ is measured at $\phi_{\min }$ [see Fig. 4(c)], in agreement with the value $\Delta J_{\min }=0.85$ expected for an optimally squeezed state (within the one-axis twisting dynamics). We report in Fig. 4(d) the corresponding distribution $\mathcal{C}_{\mathbf{n}}$. The measured $\mathcal{C}_{\mathbf{n}}$ takes its maximum for $\theta=\pi / 2$ and $\phi=\phi_{\min }$, i.e., along the squeezed quadrature direction. This maximum gives a value for the concurrence $\mathcal{C}=0.058(6)$, in agreement with the expected value of 0.055 .

Our measurements can be used to check the direct link between quadrature squeezing and nonclassical pairwise correlations [57]. Indeed, for the states reached via the one-axis twisting dynamics, one expects the concurrence to be expressed in terms of the minimum spin projection uncertainty, as

$$
\mathcal{C}=\frac{1-2 \Delta J_{\min }^{2} / J}{2 J-1} .
$$

From the measured projection quadrature, we calculate a value of 0.053(5) for the right-hand side of Eq. (5), in agreement with the direct measurement of the concurrence.

\section{PROBING ENTANGLEMENT VIA THE SUBSYSTEM ENTROPY}

So far, we studied the entanglement of $2 J$-qubit states via the nonclassical character of their qubit pairs. In this section, we access entanglement more directly, by probing whether a given state of the spin $J=8$ is separable with respect to the $14 \mid 2$ partition performed by the photon absorption. For this, we use the fact that for a separable state, the global state is more disordered than its parts [58]. More precisely, we quantify disorder via the Rényi entropy of infinite order (also called the min-entropy), defined as [59]

$$
S_{\infty}(\rho)=-\ln \lambda_{\max }(\rho),
$$

where $\lambda_{\max }$ is the maximum eigenvalue of the density matrix $\rho$. This eigenvalue corresponds to the maximum possible overlap of $\rho$ with a pure state. To reveal entanglement within a state $\rho$ of the collective spin $J$, it is thus sufficient to show that the entropy of the reduced pair state $\rho_{\text {pair }}$ is strictly higher than that of the original state $\rho$, i.e., if the conditional entropy satisfies [58]

$$
S_{\infty}(14 \mid 2) \equiv S_{\infty}(\rho)-S_{\infty}\left(\rho_{\text {pair }}\right)<0 .
$$

\section{A. Entanglement of the W state}

The evaluation of the pair state entropy $S_{\infty}\left(\rho_{\text {pair }}\right)$ is based on the tomography of the pair density matrix [60]. Full information on the density matrix is contained in the Husimi function $Q_{\text {pair }}(\mathbf{n})$. We fit the measured Husimi function by a spherical harmonic expansion

$$
Q_{\text {pair }}(\mathbf{n})=\frac{1}{3}+\sqrt{\frac{4 \pi}{3}} \sum_{\ell=1}^{2} \sum_{m=-\ell}^{\ell} \lambda_{\ell, m} Y_{\ell}^{m}(\mathbf{n})
$$

and infer the density matrix as

$$
\rho_{\text {pair }}=\frac{1}{3} \mathbb{1}+\sum_{m=-1}^{1} \lambda_{1, m} \mathcal{L}_{m}+\sum_{m=-2}^{2} \lambda_{2, m} \mathcal{Q}_{m},
$$

where the $\mathcal{L}_{m}$ and $\mathcal{Q}_{m}$ matrices correspond to the $L=1$ angular momentum components and quadrupole moments, respectively (see Appendix B). 

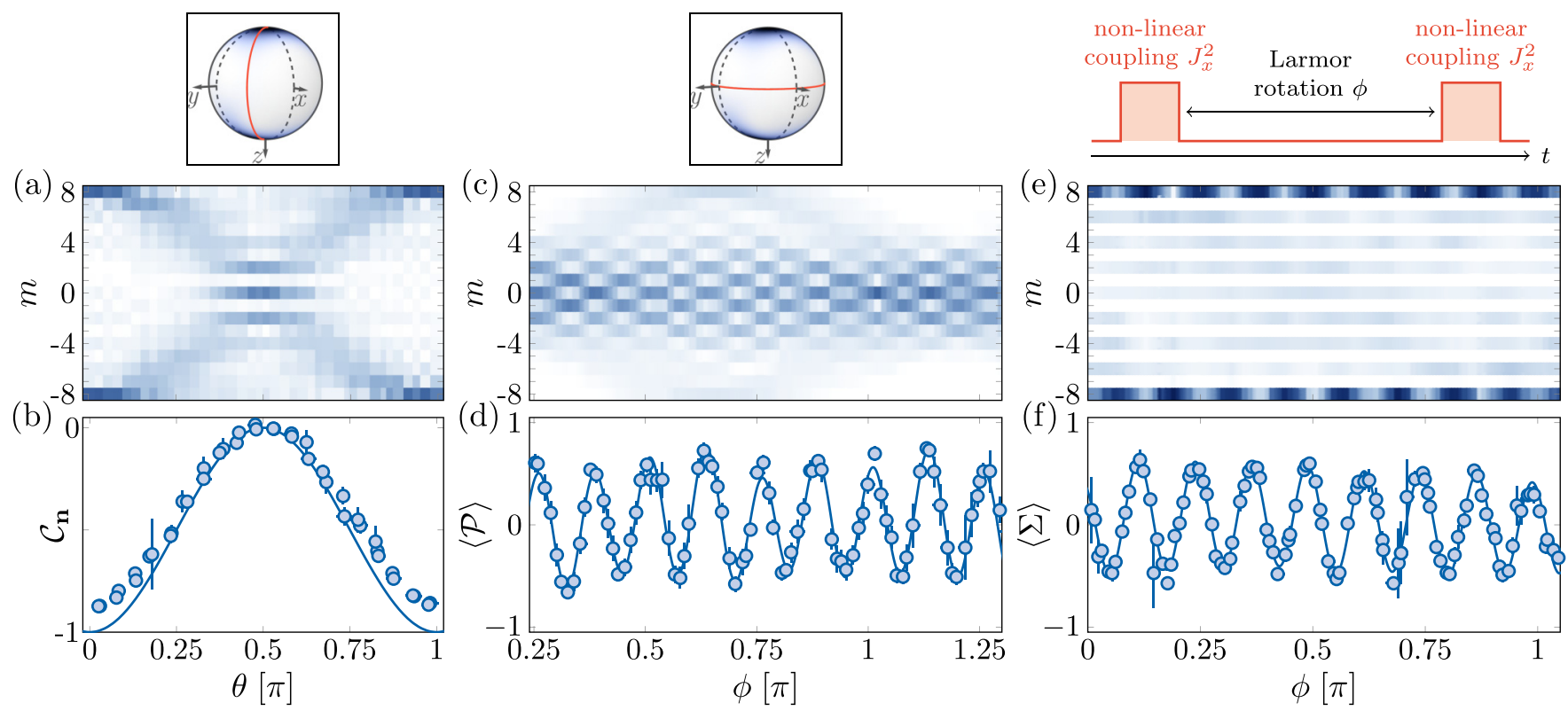

FIG. 5. Characterization of entanglement in a Schrödinger cat state. (a) Measured spin projection probabilities $\Pi_{m}$ for a cat state, as a function of the polar angle $\theta$. The azimuthal angle $\phi=0.86(5)$ rad is chosen such that the two coherent-state Husimi functions destructively interfere for odd $m$ values around $\theta=\pi / 2$. (b) Distribution $\mathcal{C}_{\mathbf{n}}$ inferred from the probabilities shown in (a) (blue circles). The solid line is the expected variation for a perfect cat state. (c) Projection probabilities $\Pi_{m}$ measured along equatorial directions $(\theta=\pi / 2)$ parametrized by the azimuthal angle $\phi$. (d) Evolution of the mean parity $\langle\mathcal{P}\rangle$ deduced from (c). (e) Projection probabilities $\Pi_{m}$ measured after a Larmor rotation of angle $\phi$ followed by a second nonlinear evolution. (f) Evolution of the mean sign of even projections $\langle\Sigma\rangle$ deduced from (e). The solid lines in (d) and (f) are fits with a Fourier series.

We apply this protocol to the $\mathrm{W}$ state, taking into account the slight variation of the Husimi function $Q_{\text {pair }}(\mathbf{n})$ with respect to the azimuthal angle $\phi$ in the prepared state [50]. We infer a density matrix

$$
\rho_{\text {pair }} \simeq\left(\begin{array}{ccc}
0.88 & 0.01+0.05 i & -0.01-0.01 i \\
0.01-0.05 i & 0.12 & 0.01 i \\
-0.01+0.01 i & -0.01 i & 0
\end{array}\right),
$$

with typically $1 \%$ statistical uncertainty. The reconstructed density matrix matches well the expected one

$$
\rho_{\text {pair }}=\left(\begin{array}{ccc}
7 / 8 & 0 & 0 \\
0 & 1 / 8 & 0 \\
0 & 0 & 0
\end{array}\right) \text {. }
$$

Diagonalization of the reconstructed density matrix gives a maximum eigenvalue $\lambda_{\max }\left(\rho_{\text {pair }}\right)=0.882(5)$.

We now consider the global spin- $J$ state. The projection probability $\Pi_{-J+1}=0.91(1)$ with the Dicke state $|m=-J+1\rangle$ provides a lower bound on the maximum overlap $\lambda_{\max }(\rho)$ with pure states.

Combining these results together, we obtain

$$
S_{\infty}(14 \mid 2)<-0.03(1) .
$$

Its negative value shows that the prepared state is not separable with respect to a $14 \mid 2$ partition, and is thus entangled.

\section{B. Entanglement of a Schrödinger cat state}

We now consider the case of a Schrödinger cat state, for which the effect of the 14|2 partition is more striking. Schrödinger cat states, which constitute archetypal states with highly nonclassical properties, have been realized in different types of experiments [30,56,61-77].

The cat state considered here is the coherent superposition of two quasiclassical spin states $|m= \pm J\rangle$ [78]. To produce it, we use the one-axis twisting dynamics discussed above, with a stronger nonlinear coupling $\chi=2 \pi \times 1.25 \mathrm{MHz}$ and a reduced magnetic field $B=53.7(1) \mathrm{mG}$. After showing quadrature squeezing at short times $(t \sim 10 \mathrm{~ns})$, the spin quadratures collapse to a featureless spin distribution, before a revival at a time $t_{\mathrm{cat}}=\pi /(2 \chi)=200 \mathrm{~ns}$, at which the system forms a coherent superposition of stretched states $|m= \pm J\rangle[56]$.

In Fig. 5(a), we show the measured probabilities $\Pi_{m}(\mathbf{n})$ for various polar angles $\theta$ with a fixed azimuthal angle $\phi$. For $\theta=0$, we confirm the dominant population of the two stretched states, with $\Pi_{-J}=0.38(2)$ and $\Pi_{J}=0.42(2)$. When varying $\theta$, the distribution is a superposition of the contributions of each of the two coherent states forming the cat state. Interestingly, we observe an interference between the two distributions when they overlap, i.e., for $\theta \simeq \pi / 2$. As shown in Fig. 5(c), the interference pattern depends on the azimuthal angle $\phi$, with an alternation between even- and odd- $m$ projections of period $2 \pi /(2 J)$ [79].

We first test whether a qubit pair extracted from this state features nonclassical behavior. We expect the distribution $\mathcal{C}_{\mathbf{n}}$ to be rotationally invariant around $z$ and thus study its variation with the polar angle $\theta$ in Fig. 5(b) [80]. Our measurements are consistent with $\mathcal{C}_{\mathbf{n}}<0$ for all angles $\theta$, showing that the reduced two-qubit state is classical. This measurement highlights the well-known property of this state that any of its subsystems is classical. 
We now extend the tomography protocol to the cat state and obtain the reduced two-body density matrix

$$
\rho_{\text {pair }} \simeq\left(\begin{array}{ccc}
0.46 & -0.01 i & -0.03+0.05 i \\
0.01 i & 0.05 & -0.01 i \\
-0.03-0.05 i & 0.01 i & 0.49
\end{array}\right),
$$

which we compare with the expected matrix

$$
\rho_{\text {pair }}=\left(\begin{array}{ccc}
1 / 2 & 0 & 0 \\
0 & 0 & 0 \\
0 & 0 & 1 / 2
\end{array}\right)
$$

obtained for a perfect cat state. We compute the maximum eigenvalue $\lambda_{\text {max }}\left(\rho_{\text {pair }}\right)=0.53(1)$ of the reconstructed matrix.

In order to reveal entanglement in the prepared state, we evaluate its overlap with perfect cat states $|\operatorname{cat}(\alpha)\rangle=$ $\left(|m=-J\rangle+e^{i \alpha}|m=J\rangle\right) / \sqrt{2}$, which constitute a family of pure quantum states. The simple form of these states in the Dicke basis allows us to express the overlap with a state $\rho$ as

$$
\mathcal{O}_{\alpha}=\frac{\rho_{-J,-J}+\rho_{J, J}+2 \operatorname{Re}\left(\rho_{-J, J} e^{i \alpha}\right)}{2},
$$

where the diagonal elements $\rho_{m, m}$ correspond to the spin projection probabilities $\Pi_{m}$. The overlap $\mathcal{O}_{\alpha}$ takes its maximum value $\mathcal{O}$ for $\alpha=-\arg \rho_{-J, J}$, with

$$
\mathcal{O}=\frac{\Pi_{-J}+\Pi_{J}+2\left|\rho_{-J, J}\right|}{2} .
$$

We present two protocols giving a lower bound on the extremal coherence $\left|\rho_{-J, J}\right|$, both based on the measurement of an observable $A$ defined on the spin $J$. We consider its mean value in a state obtained after the cat state preparation, followed by a Larmor rotation around $z$ of angle $\phi$, as

$$
\langle A\rangle(\phi)=\sum_{m, m^{\prime}} a_{m, m^{\prime}} \rho_{m^{\prime}, m} e^{i\left(m^{\prime}-m\right) \phi} .
$$

The extremal coherence can be singled out by measuring the Fourier coefficient $A_{2 J}=\left|a_{J,-J} \rho_{-J, J}\right|$ at frequency $2 J[77,79]$. We will use observables that can take values in the interval $[-1,1]$ only, such that $\left|a_{J,-J}\right| \leqslant 1$. The coefficient $A_{2 J}$ then provides a lower bound on the extremal coherence $\left|\rho_{-J, J}\right|$.

The first observable we consider is the parity $\mathcal{P}$ of the spin projection along an equatorial direction $\mathbf{n} \perp \mathbf{e}_{z}$ an observable commonly used to characterize cat states [64,65,69,70,75-77]. We fit its oscillation, shown in Fig. 5(d), with a Fourier series, from which we get the Fourier coefficient $\mathcal{P}_{2 J}=0.26(1)$. The second observable uses a nonlinear evolution, obtained by repeating the one-axis twisting evolution used to produce the cat state [56,81-84] [see the scheme in Fig. 5(e)]. In the absence of imperfections, the system is brought to a superposition $\sin (J \phi)|m=-J\rangle+\cos (J \phi)|m=J\rangle$, which allows us to extract the maximal coherence from the projection probabilities in stretched states only. The projection probabilities measured with this protocol are shown in Fig. 5(e). In practice, we observe residual probabilities in other projection values $m$, with $m$ even only, as expected from parity symmetry. We thus use an observable $\Sigma$ defined as the sign of the spin projection on even states, with

$$
\langle\Sigma\rangle=\sum_{m \text { even }} \operatorname{sgn}(m) \Pi_{m} .
$$

Its oscillation, shown in Fig. 5(f), gives a Fourier coefficient $\Sigma_{2 J}=0.247(5)$. The advantage of the second method will become clear when we consider a more complex quantum state in the next section.

The two protocols lead to comparable estimates of the extremal coherence. Using the measured probabilities $\Pi_{ \pm J}$ quoted above, we infer a lower bound on the overlap $\mathcal{O} \geqslant$ $0.66(2)$ and thus on the eigenvalue $\lambda_{\max }(\rho)$. Together, these measurements provide a conditional entropy

$$
S_{\infty}(14 \mid 2)<-0.23(3),
$$

which proves entanglement more evidently than for the $\mathrm{W}$ state. We note that the requirement $\mathcal{O}>\lambda_{\max }\left(\rho_{\text {pair }}\right)=$ $0.53(1)$, which we used to demonstrate the nonseparability of the $14 \mid 2$ partition, is consistent with the entanglement witness $\mathcal{O}>0.5$ extensively used for cat states [25].

\section{DECOHERENCE UPON QUBIT LOSS}

We now consider the removal of a pair of qubits randomly drawn from the electronic spin, irrespective of its quantum state. For this purpose, we prepare a quantum state of interest $\rho^{\prime}$ in an excited level of angular momentum $J^{\prime}=9$, corresponding to a symmetric state of $2 J^{\prime}=18$ qubits [see Fig. 1(b)]. The spontaneous emission of a photon drives the system to the ground state $J=8$, which has two missing qubits. Since the emitted photon can carry an arbitrary polarization, the process allows for three independent quantum jumps associated with the polarizations $\mathbf{e}_{-}, \mathbf{e}_{z}, \mathbf{e}_{+}$, with $\mathbf{e}_{ \pm}=$ $\left(\mathbf{e}_{x} \pm i \mathbf{e}_{y}\right) / \sqrt{2}$. The ground-state density matrix then reads

$$
\rho=\sum_{\mathbf{e}_{u}=\mathbf{e}_{-}, \mathbf{e}_{z}, \mathbf{e}_{+}}\left\langle\mathbf{e}_{u}\left|\rho^{\prime}\right| \mathbf{e}_{u}\right\rangle,
$$

which can be simply written as

$$
\rho=\operatorname{Tr}_{2} \rho^{\prime},
$$

corresponding to the loss of an arbitrary qubit pair.

\section{A. Robustness of pairwise quantum correlations}

We first investigate the effect of particle loss on a $\mathrm{W}$ state prepared in an excited electronic level of angular momentum $J^{\prime}=J+1$, coupled to the ground state with an optical transition of wavelength $626 \mathrm{~nm}$. To produce the state $\mid m^{\prime}=$ $\left.-J^{\prime}+1\right\rangle$ in the excited level, we start in the coherent state $|m=-J\rangle$ of the lowest energy manifold and use $\pi$ polarized resonant light to couple the system to the desired state [see Fig. 6(a)]. As shown in Fig. 6(b), we monitor the Rabi oscillation via the atom recoil upon light absorption. The comparison with a master equation model taking into account spontaneous emission during the Rabi flopping allows us to estimate a fidelity of 0.98 for a pulse duration $t_{\text {pulse }} \simeq 62 \mathrm{~ns}-$ the excited state lifetime being $\tau_{\text {exc }} \simeq 1.2 \mu \mathrm{s}$ [85].

Following the light pulse, we wait for spontaneous emission to occur before measuring the spin state in the ground level. We observe significant populations only in the states $|m=-J\rangle$ and $|m=-J+1\rangle$, as expected from the selection rule $\left|m^{\prime}-m\right| \leqslant 1$. The state $|m=-J+1\rangle$ is dominantly populated, showing that, in most cases, the $|\uparrow\rangle$ excitation of the $\mathrm{W}$ state is not removed upon the loss of a qubit pair. The 
(a)

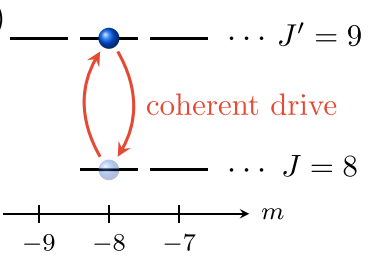

(b)

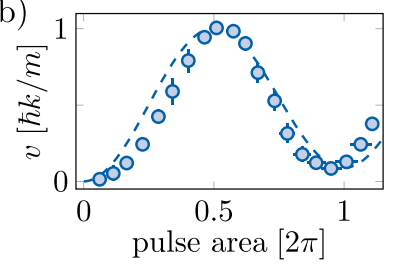

(c)

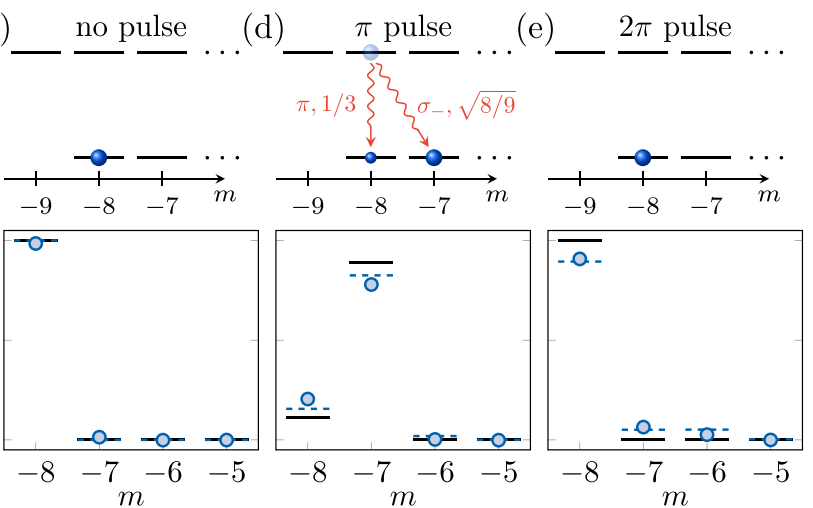

FIG. 6. Loss of a qubit pair in a W state. (a) Scheme for the preparation of the W state in the excited electronic level. (b) Evolution of the mean atom velocity acquired due to the photon absorption recoil, as a function of the light pulse duration. The dashed line is a model taking into account spontaneous emission during the pulse. (c)-(e) Top panels: expected states, with a scheme of spontaneous emission in (d) showing the Clebsch-Gordan coefficients for the two possible quantum jumps. Bottom panels: spin projection probabilities in the absence of the resonant light pulse (c), for a $\pi$ pulse (d), and for a $2 \pi$ pulse (e). The solid lines are the probabilities expected for a perfect $\mathrm{W}$ state, while the dashed lines use the same model as in (b).

projection probabilities, shown in Fig. 6(d), are close to the expected values $\Pi_{-J+1}=1 /(J+1)$ and $\Pi_{-J}=1-\Pi_{-J+1}$, with a residual difference mostly explained by the imperfect state preparation.

(a)

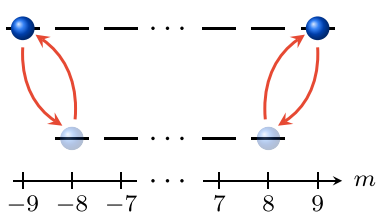

(b)

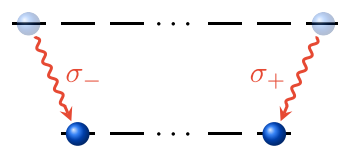

spontaneous emission
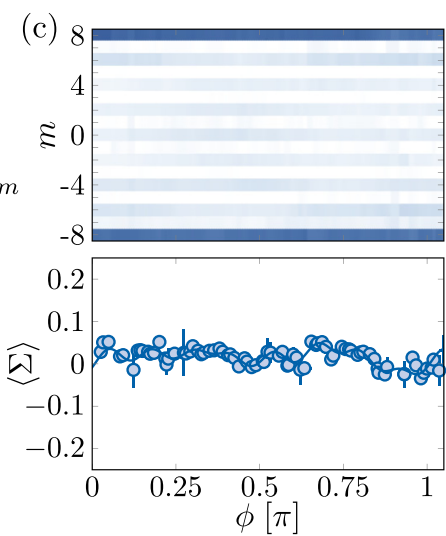

The nonclassicality of qubit pairs in the final state is probed via the distribution $\mathcal{C}_{\mathbf{n}}$ introduced in Sec. III A. We remind the reader that $\mathcal{C}_{\mathbf{n}}$ is obtained from the spin projection probabilities along $\mathbf{n}$. Since its maximum value is expected to be reached along $z$, we only consider projections along this direction, and obtain $\mathcal{C}_{z}=0.104(3)$. This value provides a lower bound on the qubit pair concurrence, expected to be $\mathcal{C}=1 /(J+1) \simeq 0.111$ in the initial state. The proximity of the initial state concurrence and the measured one after decay illustrates that losing qubits does not alter nonclassicality of the remaining qubit pairs [24].

\section{B. Fragility of macroscopic coherence}

We contrast this behavior with the fragility of entanglement in coherent superpositions of states distant in phase space [86].

We consider two examples, namely, a cat state $\left|\psi_{1}\right\rangle=$ $\left(\left|m^{\prime}=-J^{\prime}\right\rangle+\left|m^{\prime}=J^{\prime}\right\rangle\right) / \sqrt{2}$ and the superposition $\left|\psi_{2}\right\rangle=$ $\left(\left|m^{\prime}=-J^{\prime}+1\right\rangle+\left|m^{\prime}=J^{\prime}-1\right\rangle\right) / \sqrt{2}$. Their preparation consists in producing a cat state in the ground manifold $(|m=-J\rangle+|m=J\rangle) / \sqrt{2}$ (see Sec. IV B) and then applying resonant light to couple it to the excited manifold. The state $\left|\psi_{1}\right\rangle$ is produced using an $x$-linear polarization $\mathbf{e}_{x}=\left(\mathbf{e}_{+}+\mathbf{e}_{-}\right) / \sqrt{2}$, which dominantly couples the stretched states $|m= \pm J\rangle$ to states $\left|m^{\prime}= \pm J^{\prime}\right\rangle$ [see Fig. 7(a)]. Couplings to states $\left|m^{\prime}= \pm\left(J^{\prime}-2\right)\right\rangle$ also occur, albeit with very small Clebsch-Gordan coefficients, such that these processes can be neglected [87]. The state $\left|\psi_{2}\right\rangle$ is obtained using a $z$-linear polarization [see Fig. 7(d)]. In both cases, a coherent Rabi oscillation is observed when varying the pulse duration, and the fidelity of the preparation is limited by that of the cat state in the ground level. We show in Appendix C that the coherence of the superposition is maintained during Rabi flopping, by studying the states reached after $2 \pi$ pulses.

We study the effect of qubit loss, triggered by spontaneous emission, on the superposition states $\left|\psi_{1}\right\rangle$ and $\left|\psi_{2}\right\rangle$. For the cat state $\left|\psi_{1}\right\rangle$, we only expect the population of the stretched states $|m= \pm J\rangle$ [see Fig. 7(b)]. To check the
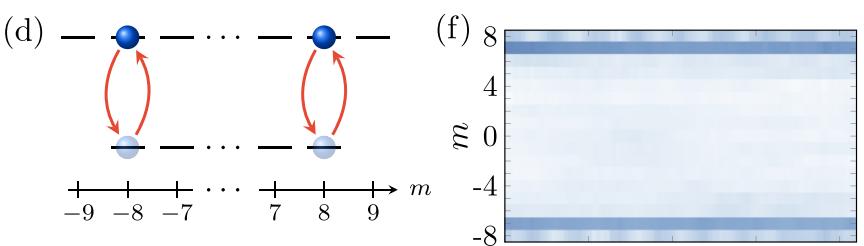

(e)

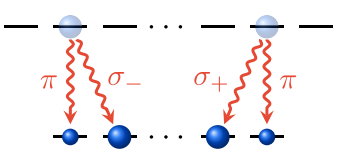

spontaneous emission

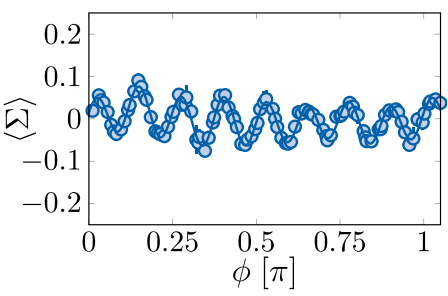

FIG. 7. Loss of a qubit pair from superposition states. (a) Preparation method for the Schrödinger cat state $\left|\psi_{1}\right\rangle$ in the excited electronic level. Given the small values of their Clebsch-Gordan coefficients, we neglect the couplings between $|m= \pm 8\rangle$ and $\left|m^{\prime}= \pm 7\right\rangle$. (b) Scheme of the subsequent spontaneous emission. (c) Top panel: spin projection probabilities measured in the $x y$ plane, as a function of the azimuthal angle $\phi$. Bottom panel: The corresponding sign observable $\langle\Sigma\rangle$, together with a fit with a Fourier series. The $y$-axis range has been reduced compared with Fig. 5(f) to highlight the absence of oscillation. (d)-(f) show the same information for the superposition state $\left|\psi_{2}\right\rangle=\left(\left|m^{\prime}=-8\right\rangle+\right.$ $\left.\left|m^{\prime}=8\right\rangle\right) / \sqrt{2}$. 
coherence between them, we measure the sign observable $\langle\Sigma\rangle$ as a function of the azimuthal angle $\phi$, as in Sec. IV B. As shown in the bottom panel of Fig. 7(c), its oscillation is completely washed out, with a measured Fourier component $\Sigma_{2 J}=0.006(10)$, indicating an absence of coherence. For the superposition state $\left|\psi_{2}\right\rangle$, we observe dominant projection probabilities in the states $|m= \pm(J-1)\rangle$, corresponding to the spontaneous emission of a $\sigma_{\mp}$ polarized photon, respectively [see Fig. 7(e)]. We do not measure any significant variation of these probabilities with the azimuthal angle $\phi$, excluding coherence between them. We also measure residual projection probabilities in the stretched states $|m= \pm J\rangle$, which occur via the spontaneous emission of a $\pi$ polarized photon. The advantage of the sign observable $\Sigma$ becomes clear here: It allows one to test the coherence between the states $|m= \pm J\rangle$, without being perturbed by the atoms populating odd- $m$ states. The measured probabilities in stretched states coherently oscillate as a function of the angle $\phi$ [see Fig. 7(f)]. More quantitatively, the sign observable, which involves even $m$ only, evolves with a Fourier component $\Sigma_{2 J}=0.024(1)$.

The complete loss of coherence when starting in the cat state $\left|\psi_{1}\right\rangle$ can be interpreted as follows. The spontaneous decay involves two orthogonal polarizations, with a $\sigma_{+}$polarized photon emitted when starting in the component $\left|m^{\prime}=-J^{\prime}\right\rangle$, while a $\sigma_{-}$polarized photon is associated with the decay of the state $\left|m^{\prime}=J^{\prime}\right\rangle$ [see Fig. 7(b)]. The photon polarization thus holds complete which path information on the spin state polarization - a term referring to Einstein's version of the double-slit interference experiment [88,89]. In this case, the coherence between the different paths is erased after spontaneous emission.

For the state $\left|\psi_{2}\right\rangle$, the most probable quantum jumps correspond to the emission of $\sigma_{+}$and $\sigma_{-}$polarized photons, which carry information about the state polarization [see Fig. 7(e)]. In contrast, the quantum jump associated with the emission of a $\pi$ polarized photon does not give this information, which explains the residual coherence. The measured Fourier coefficient $\Sigma_{2 J}$ corresponds to $9.7(5) \%$ of the value measured in the absence of the excitation. This reduction is consistent with the probability $1 /(J+1) \simeq 11.1 \%$ of scattering a $\pi$ polarized photon for the considered state, showing that this channel fully preserves coherence.

\section{SUMMARY AND OUTLOOK}

In this paper, we show that the $2 J$-qubit ensemble associated with an atomic electronic spin $J$ can be partitioned via the optical coupling to an excited level $J^{\prime}=J-1$. Among these qubits, $2 J-2$ of them constitute the excited level, and the remaining two are annihilated by the absorbed photon, in a state defined by the light polarization. We investigate this process using atomic dysprosium and use it to probe entanglement in nonclassical states of spin $J=8$. We fully characterize the nonclassical character of its reduced two-qubit state and study the increase of entropy upon partition as a smoking gun for entanglement.

In a second set of experiments, we consider the partition of an angular momentum $J^{\prime}=J+1$ of an excited electronic state. There, a random qubit pair is extracted by spontaneous

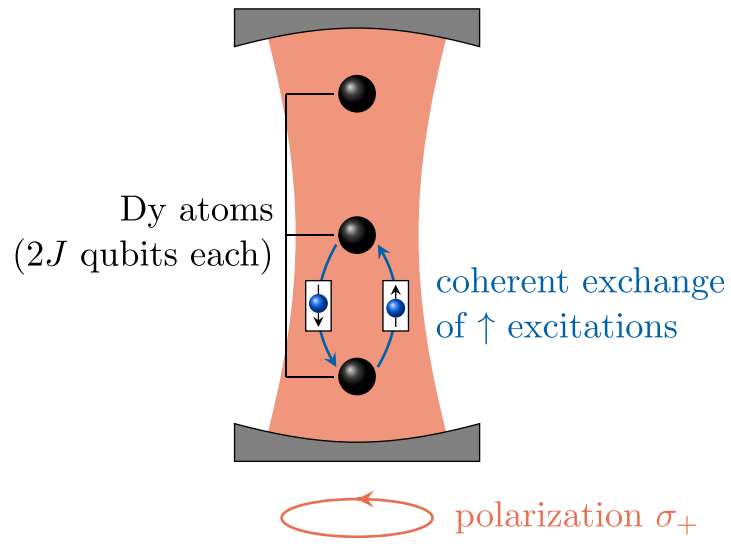

FIG. 8. Proposed scheme for entangling several Dy atoms in an optical resonator. An off-resonant optical cavity in the strongcoupling regime couples an ensemble of $N$ atoms together. For $\sigma_{+}$ polarized cavity light, the total spin projection along $z$ is conserved, and the cavity mediates the coherent exchange of $\uparrow$ qubit excitations between atoms. Such couplings can be used to stabilize a W state, with a single $\uparrow$ excitation symmetrically shared between the $N \times(2 J)$ qubits.

emission towards the ground state $J$. We show that nonclassical pairwise correlations are robust to particle loss. In contrast, we observe that coherent superpositions of states distant in phase space are very fragile.

In this paper, the study of light-spin interaction is limited to measurements of the electronic spin. A first extension would be to collect the spontaneously emitted photon, whose polarization is entangled with the electronic spin, as for experiments performed with trapped ions, atoms in optical cavities, or solid-state qubits [90-93]. One would thus explicitly access the which path information carried by the photon upon spontaneous emission of a Schrödinger cat state. More generally, the photon would allow one to couple qubit pairs from the electronic spin $J=8$ to "flying qubits," which could then be manipulated to entangle distant atoms [94], and generalize quantum communication schemes to a mesoscopic degree of freedom [95].

Another interesting perspective would be to place the atomic gas in an optical cavity. The electronic spin $J$ of a single atom would be coherently coupled to the cavity light mode, leading to a compound light-spin object [96]. For an atomic ensemble, the cavity light would also couple the electronic spins together, similarly to standard ensembles of spin-1/2 atoms coupled to optical cavities [4,97,98]. For a set of $N$ dysprosium atoms - each hosting $2 J$ qubits - the size of the Hilbert space would be $(2 J+1)^{N}$, much smaller than the size $2^{2 J N}$ for the same number of qubits realized with spin$1 / 2$ atoms. This favorable scaling will mitigate decoherence effects associated with, for example, particle loss.

To be more concrete, we show in Fig. 8 an example of an application, with an ensemble of $N$ atoms coupled to $\sigma_{+}$polarized cavity light. The light mediates the coherent exchange of $|\uparrow\rangle$ excitations among the atoms, which could serve to stabilize a W state with one excitation symmetrically shared among $N \times(2 J)$ qubits. Such many-body entangled states could feature a strong quantum enhancement of magnetic 
sensitivity $[99,100]$ or serve as a playground for studies of decoherence.

\section{ACKNOWLEDGMENT}

This work is supported by the European Union (Grant No. TOPODY 756722 from the European Research Council). We thank Jean Dalibard for stimulating discussions.

\section{APPENDIX A: DEVIATION FROM $z$ ROTATION SYMMETRY IN THE W AND CAT STATES}

The $\mathrm{W}$ state $|m=-J+1\rangle$ is invariant upon rotations around $z$, such that all observables should depend on the polar angle $\theta$ only. In practice, the state prepared close to the $\mathrm{W}$ state is not perfectly rotationally symmetric, because
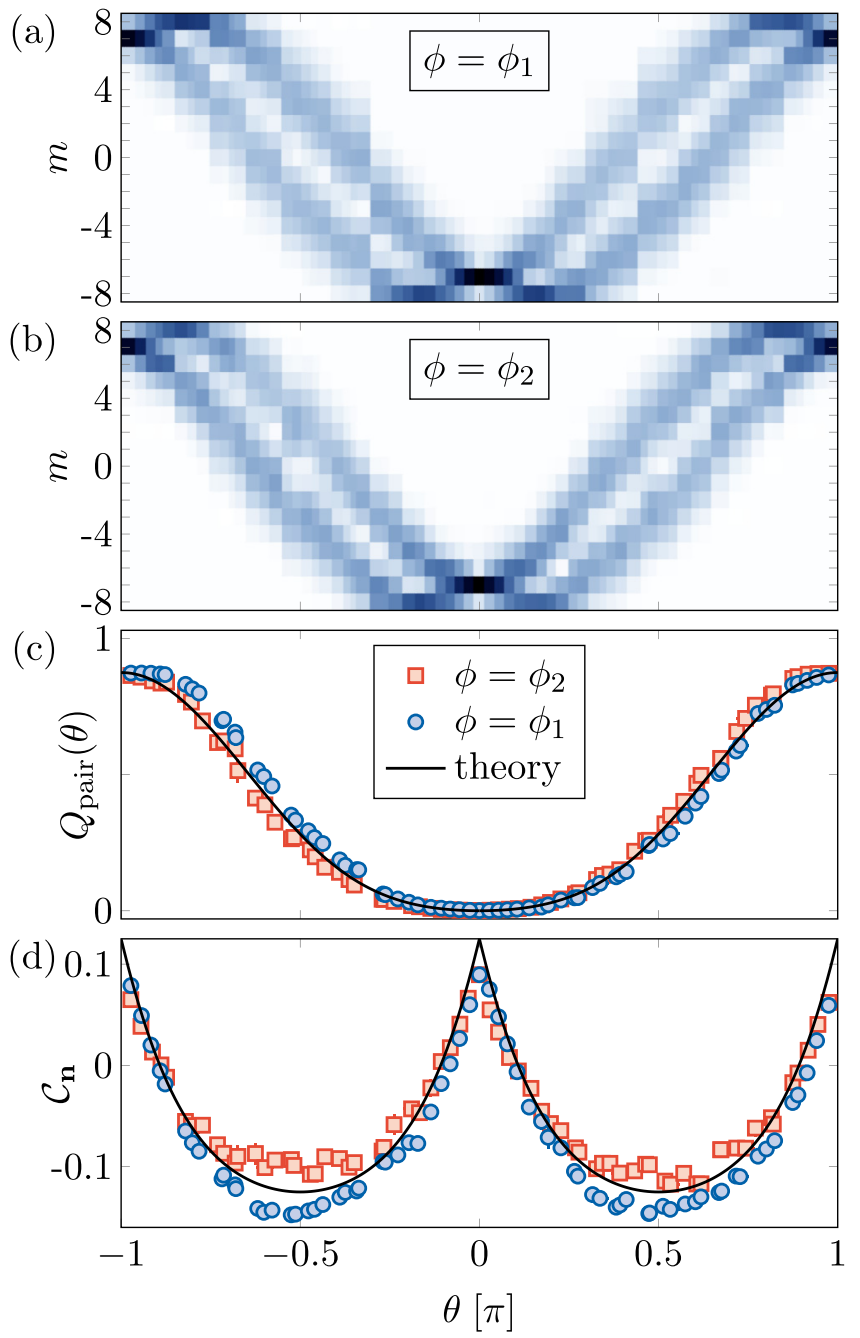

FIG. 9. Deviation from $z$ rotation symmetry in the prepared $\mathrm{W}$ state. (a) and (b) Projection probabilities $\Pi_{m}$ as a function of the polar angle $\theta$, for $\phi_{1}=0.36(5) \mathrm{rad}$ and $\phi_{2}=\phi_{1}-\pi / 2$. (c) Pair Husimi functions $Q_{\text {pair }}$ inferred from the (a) and (b) data (blue circles and red squares, respectively). The error bars represent the statistical uncertainty from a bootstrap random sampling analysis. The line corresponds to the expected variation for the $\mathrm{W}$ state. (d) Distribution $\mathcal{C}_{\mathbf{n}}$ as a function of $\theta$. The two azimuthal angles $\phi_{1}$ and $\phi_{2}$ are chosen to minimize and maximize the measured $\mathcal{C}_{\mathbf{n}}$, respectively.
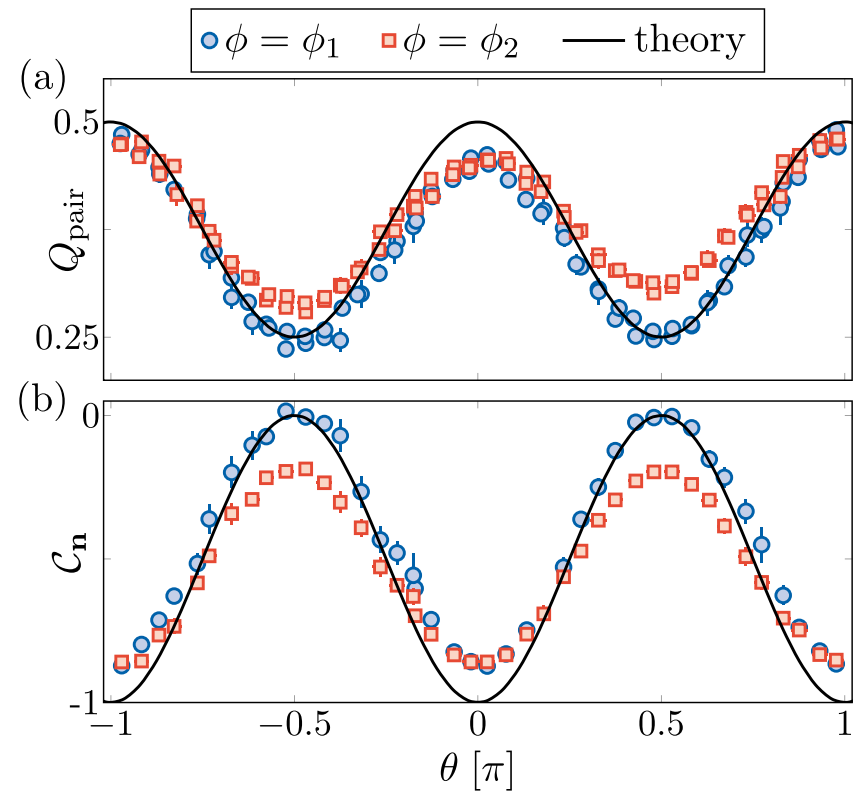

FIG. 10. Deviation from $z$ rotation symmetry in the prepared Schrödinger cat state. (a) Pair Husimi functions $Q_{\text {pair }}$ as a function of the polar angle $\theta$, for $\phi_{1}=3.3(1) \mathrm{rad}$ and $\phi_{2}=\phi_{1}-\pi / 2$ (blue circles and red squares, respectively). The line corresponds to the expected variation for a perfect cat state. (b) Distribution $\mathcal{C}_{\mathbf{n}}$ as a function of $\theta$ deduced from the data in (a).

of a residual coherent admixture with other Dicke states. We measure a small $\phi$ variation of the measured probability distributions $\Pi_{m}(\mathbf{n})$, as well as the pair Husimi function $Q_{\text {pair }}$ and distribution $\mathcal{C}_{\mathbf{n}}$ deduced from them. We show in Fig. 9 the
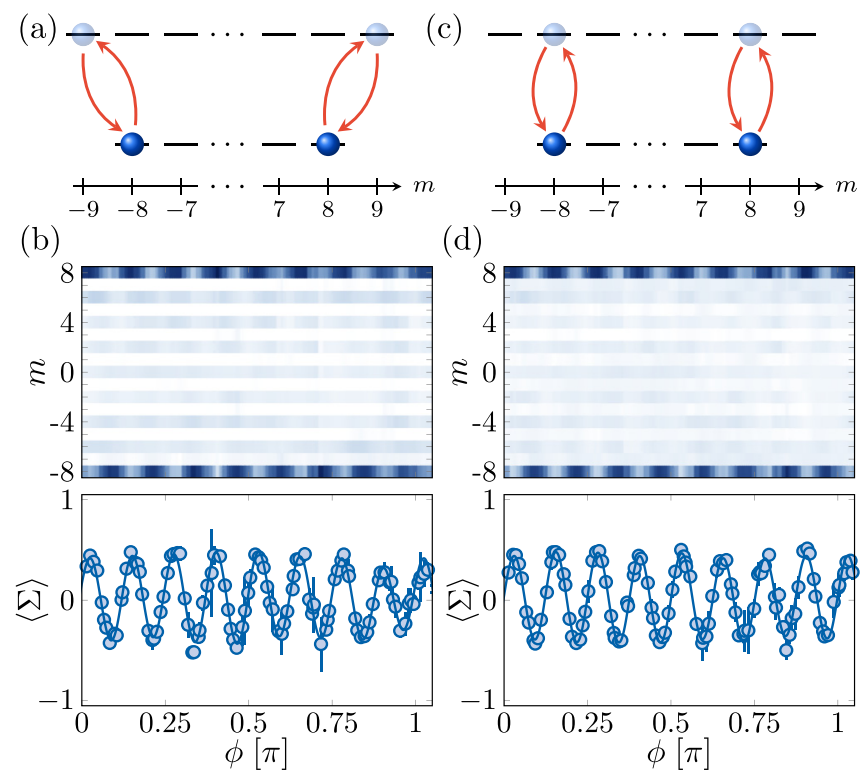

(d)

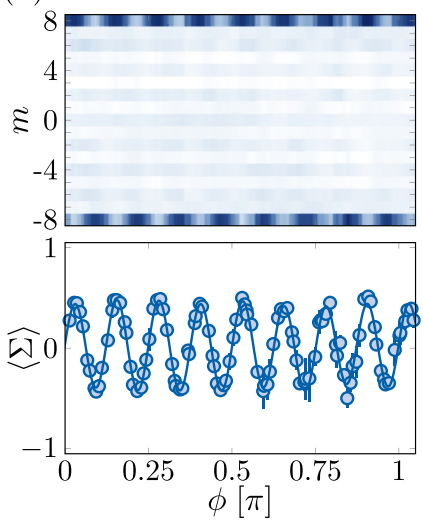

FIG. 11. (a) Scheme of the $2 \pi$ Rabi oscillation starting in a Schrödinger cat state of the electronic ground level, for an $x$ polarized laser excitation. (b) Top panel: spin projection probabilities measured in the $x y$ plane, as a function of the azimuthal angle $\phi$. Bottom panel: the corresponding sign observable $\langle\Sigma\rangle$, together with a fit with a Fourier series. (c) and (d) show the same information for a $z$-polarized laser excitation. 
measured data for two azimuthal angles $\phi_{1}=0.36(5) \mathrm{rad}$ and $\phi_{2}=\phi_{1}-\pi / 2$, for which $\mathcal{C}_{\mathbf{n}}$ is minimized and maximized, respectively. The data shown in Fig. 3 of the main text correspond to an average over $\phi$, the error bars taking into account this dispersion.

The cat state $|m=-J\rangle+|m=J\rangle$ is not rotationally invariant. Yet, its reduced two-body density matrix, given by Eq. (8), is invariant such that the pair Husimi function $Q_{\text {pair }}$ and distribution $\mathcal{C}_{\mathbf{n}}$ should depend on $\theta$ only. As for the $\mathrm{W}$ state, we measure a slight variation of these quantities with $\phi$, as shown in Fig. 10. Since we focus on extracting the concurrence from the maximum of $\mathcal{C}_{\mathbf{n}}$, we show in the main text the data measured for an azimuthal angle $\phi_{1}=3.3(1) \mathrm{rad}$ that maximizes $\mathcal{C}_{\mathbf{n}}$.

\section{APPENDIX B: SPIN-1 TOMOGRAPHY USING THE PAIR HUSIMI FUNCTION}

The Husimi function of a spin-1 quantum state $\rho$ expands on the spherical harmonics $Y_{\ell}^{m}$ with $\ell=1,2$ and $|m| \leqslant \ell$, as written in Eq. (6). This linear decomposition allows us to retrieve the density matrix $\rho$, as given by Eq. (7), where we introduce the operators

$$
\begin{gathered}
\mathcal{L}_{0}=L_{z}, \\
\mathcal{L}_{ \pm 1}=\mp\left(L_{x} \pm i L_{y}\right) / \sqrt{2},
\end{gathered}
$$

$$
\begin{gathered}
\mathcal{Q}_{0}=\sqrt{\frac{5}{3}}\left(3 L_{z}^{2}-2\right), \\
\mathcal{Q}_{ \pm 1}=\mp \sqrt{\frac{5}{2}}\left[\left(L_{x} \pm i L_{y}\right) L_{z}+L_{z}\left(L_{x} \pm i L_{y}\right)\right], \\
\mathcal{Q}_{ \pm 2}=\sqrt{\frac{5}{2}}\left(L_{x} \pm i L_{y}\right)^{2} .
\end{gathered}
$$

\section{APPENDIX C: COHERENCE OF SUPERPOSITION STATES DURING RABI FLOPPING}

The preparation of superposition states in the excited electronic state, as studied in Sec. V B, uses coherent Rabi oscillations, starting in a Schrödinger cat state of the ground electronic level $(|m=-J\rangle+|m=J\rangle) / \sqrt{2}$. To check that coherence is maintained during the Rabi oscillation, we study it after a $2 \pi$ excitation, by measuring the oscillation of the sign observable $\langle\Sigma\rangle$.

As shown in Fig. 11, we find that the coherence $\left|\rho_{-J, J}\right|$, estimated by the Fourier component $\Sigma_{2 J}$, is reduced to $0.202(2)$ $[0.211(6)]$ for the $x$-polarized (z-polarized) excitation, i.e., above $80 \%$ of the value obtained with no Rabi pulse. These measurements confirm that coherence is preserved during the Rabi oscillation.
[1] S. J. Freedman and J. F. Clauser, Experimental Test of Local Hidden-Variable Theories, Phys. Rev. Lett. 28, 938 (1972).

[2] A. Aspect, J. Dalibard, and G. Roger, Experimental Test of Bell's Inequalities Using Time-Varying Analyzers, Phys. Rev. Lett. 49, 1804 (1982).

[3] L. Amico, R. Fazio, A. Osterloh, and V. Vedral, Entanglement in many-body systems, Rev. Mod. Phys. 80, 517 (2008).

[4] L. Pezzè, A. Smerzi, M. K. Oberthaler, R. Schmied, and P. Treutlein, Quantum metrology with nonclassical states of atomic ensembles, Rev. Mod. Phys. 90, 035005 (2018).

[5] A. Sørensen, L.-M. Duan, J. I. Cirac, and P. Zoller, Many-particle entanglement with Bose-Einstein condensates, Nature (London) 409, 63 (2001).

[6] A. S. Sørensen and K. Mølmer, Entanglement and Extreme Spin Squeezing, Phys. Rev. Lett. 86, 4431 (2001).

[7] J. Esteve, C. Gross, A. Weller, S. Giovanazzi, and M. Oberthaler, Squeezing and entanglement in a Bose-Einstein condensate, Nature (London) 455, 1216 (2008).

[8] M. F. Riedel, P. Böhi, Y. Li, T. W. Hänsch, A. Sinatra, and P. Treutlein, Atom-chip-based generation of entanglement for quantum metrology, Nature (London) 464, 1170 (2010).

[9] C. Gross, T. Zibold, E. Nicklas, J. Estève, and M. K. Oberthaler, Nonlinear atom interferometer surpasses classical precision limit, Nature (London) 464, 1165 (2010).

[10] P. Hyllus, W. Laskowski, R. Krischek, C. Schwemmer, W. Wieczorek, H. Weinfurter, L. Pezzé, and A. Smerzi, Fisher information and multiparticle entanglement, Phys. Rev. A 85, 022321 (2012).
[11] G. Tóth, Multipartite entanglement and high-precision metrology, Phys. Rev. A 85, 022322 (2012).

[12] J.-W. Pan, Z.-B. Chen, C.-Y. Lu, H. Weinfurter, A. Zeilinger, and M. Żukowski, Multiphoton entanglement and interferometry, Rev. Mod. Phys. 84, 777 (2012).

[13] G. Wendin, Quantum information processing with superconducting circuits: A review, Rep. Prog. Phys. 80, 106001 (2017).

[14] R. Blatt and D. Wineland, Entangled states of trapped atomic ions, Nature (London) 453, 1008 (2008).

[15] M. Saffman, T. G. Walker, and K. Mølmer, Quantum information with Rydberg atoms, Rev. Mod. Phys. 82, 2313 (2010).

[16] R. Horodecki, P. Horodecki, M. Horodecki, and K. Horodecki, Quantum entanglement, Rev. Mod. Phys. 81, 865 (2009).

[17] O. Gühne and G. Tóth, Entanglement detection, Phys. Rep. 474, 1 (2009).

[18] S. Hill and W. K. Wootters, Entanglement of a Pair of Quantum Bits, Phys. Rev. Lett. 78, 5022 (1997).

[19] W. K. Wootters, Entanglement of Formation of an Arbitrary State of Two Qubits, Phys. Rev. Lett. 80, 2245 (1998).

[20] F. A. Bovino, G. Castagnoli, A. Ekert, P. Horodecki, C. M. Alves, and A. V. Sergienko, Direct Measurement of Nonlinear Properties of Bipartite Quantum States, Phys. Rev. Lett. 95, 240407 (2005).

[21] S. P. Walborn, P. H. Souto Ribeiro, L. Davidovich, F. Mintert, and A. Buchleitner, Experimental determination of entanglement with a single measurement, Nature (London) 440, 1022 (2006). 
[22] C. Schmid, N. Kiesel, W. Wieczorek, H. Weinfurter, F. Mintert, and A. Buchleitner, Experimental Direct Observation of Mixed State Entanglement, Phys. Rev. Lett. 101, 260505 (2008).

[23] R. Islam, R. Ma, P. M. Preiss, M. Eric Tai, A. Lukin, M. Rispoli, and M. Greiner, Measuring entanglement entropy in a quantum many-body system, Nature (London) 528, 77 (2015).

[24] W. Dür, G. Vidal, and J. I. Cirac, Three qubits can be entangled in two inequivalent ways, Phys. Rev. A 62, 062314 (2000).

[25] G. Tóth and O. Gühne, Detecting Genuine Multipartite Entanglement with Two Local Measurements, Phys. Rev. Lett. 94, 060501 (2005).

[26] R. Lapkiewicz, P. Li, C. Schaeff, N. K. Langford, S. Ramelow, M. Wieśniak, and A. Zeilinger, Experimental non-classicality of an indivisible quantum system, Nature (London) 474, 490 (2011).

[27] S. Chaudhury, S. Merkel, T. Herr, A. Silberfarb, I. H. Deutsch, and P. S. Jessen, Quantum Control of the Hyperfine Spin of a Cs Atom Ensemble, Phys. Rev. Lett. 99, 163002 (2007).

[28] T. Fernholz, H. Krauter, K. Jensen, J. F. Sherson, A. S. Sørensen, and E. S. Polzik, Spin Squeezing of Atomic Ensembles via Nuclear-Electronic Spin Entanglement, Phys. Rev. Lett. 101, 073601 (2008).

[29] D. Gatteschi and R. Sessoli, Quantum tunneling of magnetization and related phenomena in molecular materials, Angew. Chem. Int. Ed. 42, 268 (2003).

[30] A. Facon, E.-K. Dietsche, D. Grosso, S. Haroche, J.-M. Raimond, M. Brune, and S. Gleyzes, A sensitive electrometer based on a Rydberg atom in a Schrödinger-cat state, Nature (London) 535, 262 (2016).

[31] E. Majorana, Atomi orientati in campo magnetico variabile, Nuovo Cim. 9, 43 (1932).

[32] In practice, since the light shift amplitudes strongly vary with $m$, we vary the pulse duration in the range $10-100 \mu$ s and the detuning in the range $50 \mathrm{MHz}$ to $1 \mathrm{GHz}$, in order to keep similar displacements for all $m$ states (except the dark states). For the smallest detunings, we take into account the corrections to the second-order light shifts. The uncertainties in the laser beam waist $w=40(5) \mu \mathrm{m}$ and in the excited state lifetime $\tau \simeq 11 \mu$ s [101] lead to a systematic error. We correct an overall $20 \%$ error using the constraint $\sum_{m} Q_{m}=(2 J+1) / 3$, which states that in a completely undetermined state, a symmetric qubit pair has $1 / 3$ chance to be in $|\uparrow \uparrow\rangle$.

[33] R. H. Dicke, Coherence in spontaneous radiation processes, Phys. Rev. 93, 99 (1954).

[34] K. S. Choi, A. Goban, S. B. Papp, S. J. van Enk, and H. J. Kimble, Entanglement of spin waves among four quantum memories, Nature (London) 468, 412 (2010).

[35] F. Haas, J. Volz, R. Gehr, J. Reichel, and J. Estève, Entangled states of more than 40 atoms in an optical fiber cavity, Science 344, 180 (2014).

[36] R. McConnell, H. Zhang, J. Hu, S. Ćuk, and V. Vuletić, Entanglement with negative Wigner function of almost 3,000 atoms heralded by one photon, Nature (London) 519, 439 (2015).

[37] M. Ebert, M. Kwon, T. G. Walker, and M. Saffman, Coherence and Rydberg Blockade of Atomic Ensemble Qubits, Phys. Rev. Lett. 115, 093601 (2015).

[38] J. Zeiher, P. Schauß, S. Hild, T. Macrì, I. Bloch, and C. Gross, Microscopic Characterization of Scalable Coherent Rydberg Superatoms, Phys. Rev. X 5, 031015 (2015).
[39] H. Häffner, W. Hänsel, C. F. Roos, J. Benhelm, D. Chek-alkar, M. Chwalla, T. Körber, U. D. Rapol, M. Riebe, P. O. Schmidt, C. Becher, O. Gühne, W. Dür, and R. Blatt, Scalable multiparticle entanglement of trapped ions, Nature (London) 438, 643 (2005).

[40] F. Fröwis, P. C. Strassmann, A. Tiranov, C. Gut, J. Lavoie, N. Brunner, F. Bussières, M. Afzelius, and N. Gisin, Experimental certification of millions of genuinely entangled atoms in a solid, Nat. Commun. 8, 907 (2017).

[41] Y. Pu, Y. Wu, N. Jiang, W. Chang, C. Li, S. Zhang, and L. Duan, Experimental entanglement of 25 individually accessible atomic quantum interfaces, Sci. Adv. 4, 3931 (2018).

[42] P. Facchi and S. Pascazio, Quantum Zeno Subspaces, Phys. Rev. Lett. 89, 080401 (2002).

[43] F. Schäfer, I. Herrera, S. Cherukattil, C. Lovecchio, F. S Cataliotti, F. Caruso, and A. Smerzi, Experimental realization of quantum Zeno dynamics, Nat. Commun. 5, 3194 (2014).

[44] A. Signoles, A. Facon, D. Grosso, I. Dotsenko, S. Haroche, J.-M. Raimond, M. Brune, and S. Gleyzes, Confined quantum Zeno dynamics of a watched atomic arrow, Nat. Phys. 10, 715 (2014).

[45] The W-state fidelity is mostly limited by inelastic collisions between atoms, which redistribute the spin among neighboring $m$ states.

[46] E. C. G. Sudarshan, Equivalence of Semiclassical and Quantum Mechanical Descriptions of Statistical Light Beams, Phys. Rev. Lett. 10, 277 (1963).

[47] R. J. Glauber, Coherent and Incoherent States of the Radiation Field, Phys. Rev. 131, 2766 (1963).

[48] O. Giraud, P. Braun, and D. Braun, Classicality of spin states, Phys. Rev. A 78, 042112 (2008).

[49] J. K. Korbicz, J. I. Cirac, and M. Lewenstein, Spin Squeezing Inequalities and Entanglement of $N$ Qubit States, Phys. Rev. Lett. 95, 120502 (2005).

[50] While the W state is rotationally symmetric around $z$, we observe in the prepared state a small but significant variation of the projection probabilities with the azimuthal angle $\phi$. We show in Figs. 3(b)-3(d) the $\phi$-averaged values of our measurements. The variation with $\phi$ of the measured quantities is taken into account in the error bars and is explicitly shown in Appendix A.

[51] M. Hillery, Nonclassical distance in quantum optics, Phys. Rev. A 35, 725 (1987).

[52] T.-C. Wei and P. M. Goldbart, Geometric measure of entanglement and applications to bipartite and multipartite quantum states, Phys. Rev. A 68, 042307 (2003).

[53] J. Vidal, Concurrence in collective models, Phys. Rev. A 73, 062318 (2006).

[54] M. Koashi, V. Bužek, and N. Imoto, Entangled webs: Tight bound for symmetric sharing of entanglement, Phys. Rev. A 62, 050302(R) (2000).

[55] M. Kitagawa and M. Ueda, Squeezed spin states, Phys. Rev. A 47, 5138 (1993).

[56] T. Chalopin, C. Bouazza, A. Evrard, V. Makhalov, D. Dreon, J. Dalibard, L. A. Sidorenkov, and S. Nascimbene, Quantumenhanced sensing using non-classical spin states of a highly magnetic atom, Nat. Commun. 9, 4955 (2018).

[57] X. Wang and B. C. Sanders, Spin squeezing and pairwise entanglement for symmetric multiqubit states, Phys. Rev. A 68, 012101 (2003). 
[58] R. Horodecki and M. Horodecki, Information-theoretic aspects of inseparability of mixed states, Phys. Rev. A 54, 1838 (1996).

[59] R. Konig, R. Renner, and C. Schaffner, The operational meaning of min- and max-entropy, IEEE Trans. Inf. Theory 55, 4337 (2009).

[60] V. I. Man'ko and O. V. Man'ko, Spin state tomography, J. Exp. Theor. Phys. 85, 430 (1997).

[61] C. Monroe, D. Meekhof, B. King, and D. J. Wineland, A "Schrödinger cat" superposition state of an atom, Science 272, 1131 (1996).

[62] M. Brune, E. Hagley, J. Dreyer, X. Maître, A. Maali, C. Wunderlich, J. M. Raimond, and S. Haroche, Observing the Progressive Decoherence of the "Meter" in a Quantum Measurement, Phys. Rev. Lett. 77, 4887 (1996).

[63] J. R. Friedman, V. Patel, W. Chen, S. Tolpygo, and J. E. Lukens, Quantum superposition of distinct macroscopic states, Nature (London) 406, 43 (2000).

[64] C. A. Sackett, D. Kielpinski, B. E. King, C. Langer, V. Meyer, C. J. Myatt, M. Rowe, Q. A. Turchette, W. M. Itano, D. J. Wineland, and C. Monroe, Experimental entanglement of four particles, Nature (London) 404, 256 (2000).

[65] D. Leibfried, E. Knill, S. Seidelin, J. Britton, R. B. Blakestad, J. Chiaverini, D. B. Hume, W. M. Itano, J. D. Jost, C. Langer, R. Ozeri, R. Reichle, and D. J. Wineland, Creation of a six-atom 'Schrödinger cat' state, Nature (London) 438, 639 (2005).

[66] A. Ourjoumtsev, R. Tualle-Brouri, J. Laurat, and P. Grangier, Generating optical Schrödinger kittens for quantum information processing, Science 312, 83 (2006).

[67] J. S. Neergaard-Nielsen, B. M. Nielsen, C. Hettich, K. Mølmer, and E. S. Polzik, Generation of a Superposition of Odd Photon Number States for Quantum Information Networks, Phys. Rev. Lett. 97, 083604 (2006).

[68] S. Deleglise, I. Dotsenko, C. Sayrin, J. Bernu, M. Brune, J.-M. Raimond, and S. Haroche, Reconstruction of nonclassical cavity field states with snapshots of their decoherence, Nature (London) 455, 510 (2008).

[69] T. Monz, P. Schindler, J. T. Barreiro, M. Chwalla, D. Nigg, W. A. Coish, M. Harlander, W. Hänsel, M. Hennrich, and R. Blatt, 14-Qubit Entanglement: Creation and Coherence, Phys. Rev. Lett. 106, 130506 (2011).

[70] X.-C. Yao, T.-X. Wang, P. Xu, H. Lu, G.-S. Pan, X.-H. Bao, C.-Z. Peng, C.-Y. Lu, Y.-A. Chen, and J.-W. Pan, Observation of eight-photon entanglement, Nat. Photon. 6, 225 (2012).

[71] G. Kirchmair, B. Vlastakis, Z. Leghtas, S. E. Nigg, H. Paik, E. Ginossar, M. Mirrahimi, L. Frunzio, S. M. Girvin, and R. J. Schoelkopf, Observation of quantum state collapse and revival due to the single-photon Kerr effect, Nature (London) 495, 205 (2013).

[72] C. L. Degen, F. Reinhard, and P. Cappellaro, Quantum sensing, Rev. Mod. Phys. 89, 035002 (2017).

[73] X.-L. Wang, Y.-H. Luo, H.-L. Huang, M.-C. Chen, Z.-E. Su, C. Liu, C. Chen, W. Li, Y.-Q. Fang, X. Jiang, J. Zhang, L. Li, N.-L. Liu, C.-Y. Lu, and J.-W. Pan, 18-Qubit Entanglement with Six Photons' Three Degrees of Freedom, Phys. Rev. Lett. 120, 260502 (2018).

[74] E. K. Dietsche, A. Larrouy, S. Haroche, J. M. Raimond, M. Brune, and S. Gleyzes, High-sensitivity magnetometry with a single atom in a superposition of two circular Rydberg states, Nat. Phys. 15, 326 (2019).

[75] C. Song, K. Xu, H. Li, Y.-R. Zhang, X. Zhang, W. Liu, Q. Guo, Z. Wang, W. Ren, J. Hao, H. Feng, H. Fan, D. Zheng, D.-W. Wang, H. Wang, and S.-Y. Zhu, Generation of multicomponent atomic Schrödinger cat states of up to 20 qubits, Science $\mathbf{3 6 5}$, 574 (2019).

[76] A. Omran, H. Levine, A. Keesling, G. Semeghini, T. T. Wang, S. Ebadi, H. Bernien, A. S. Zibrov, H. Pichler, S. Choi, J. Cui, M. Rossignolo, P. Rembold, S. Montangero, T. Calarco, M. Endres, M. Greiner, V. Vuletić, and M. D. Lukin, Generation and manipulation of Schrödinger cat states in Rydberg atom arrays, Science 365, 570 (2019).

[77] K. X. Wei, I. Lauer, S. Srinivasan, N. Sundaresan, D. T. McClure, D. Toyli, D. C. McKay, J. M. Gambetta, and S. Sheldon, Verifying multipartite entangled Greenberger-HorneZeilinger states via multiple quantum coherences, Phys. Rev. A 101, 032343 (2020).

[78] N. D. Mermin, Extreme Quantum Entanglement in a Superposition of Macroscopically Distinct States, Phys. Rev. Lett. 65, 1838 (1990).

[79] J. J. Bollinger, W. M. Itano, D. J. Wineland, and D. J. Heinzen, Optimal frequency measurements with maximally correlated states, Phys. Rev. A 54, R4649(R) (1996).

[80] In practice, we observe a small variation of the measured $\mathcal{C}_{\mathbf{n}}$ with the azimuthal angle $\phi$, discussed in Appendix A. We show in Fig. 5(b) the variation of $\mathcal{C}_{\mathbf{n}}$ with the polar angle $\theta$ with a fixed angle $\phi=3.3(1) \mathrm{rad}$, which maximizes the value of $\mathcal{C}_{\mathbf{n}}$.

[81] E. Davis, G. Bentsen, and M. Schleier-Smith, Approaching the Heisenberg Limit without Single-Particle Detection, Phys. Rev. Lett. 116, 053601 (2016).

[82] D. Linnemann, H. Strobel, W. Muessel, J. Schulz, R. J. Lewis-Swan, K. V. Kheruntsyan, and M. K. Oberthaler, Quantum-Enhanced Sensing Based on Time Reversal of Nonlinear Dynamics, Phys. Rev. Lett. 117, 013001 (2016).

[83] F. Fröwis, P. Sekatski, and W. Dür, Detecting Large Quantum Fisher Information with Finite Measurement Precision, Phys. Rev. Lett. 116, 090801 (2016).

[84] T. Macrì, A. Smerzi, and L. Pezzè, Loschmidt echo for quantum metrology, Phys. Rev. A 94, 010102(R) (2016).

[85] M. Gustavsson, H. Lundberg, L. Nilsson, and S. Svanberg, Lifetime measurements for excited states of rare-earth atoms using pulse modulation of a cw dye-laser beam, J. Opt. Soc. Am. 69, 984 (1979).

[86] C.-W. Lee and H. Jeong, Quantification of Macroscopic Quantum Superpositions within Phase Space, Phys. Rev. Lett. 106, 220401 (2011).

[87] The coupling amplitudes between $|m= \pm J\rangle$ and $\mid m^{\prime}=$ $\left.\pm\left(J^{\prime}-2\right)\right\rangle$ are $1 / \sqrt{153}$ smaller than the couplings between $|m= \pm J\rangle$ and $\left|m^{\prime}= \pm J^{\prime}\right\rangle$. When the population of $\left|m^{\prime}= \pm J^{\prime}\right\rangle$ is maximized, we expect a residual population of the states $\left|m^{\prime}= \pm\left(J^{\prime}-2\right)\right\rangle$ of $3 \%$ due to these small couplings.

[88] W. K. Wootters and W. H. Zurek, Complementarity in the double-slit experiment: Quantum nonseparability and a quantitative statement of Bohr's principle, Phys. Rev. D 19, 473 (1979).

[89] B.-G. Englert, Fringe Visibility and Which-Way Information: An Inequality, Phys. Rev. Lett. 77, 2154 (1996). 
[90] B. B. Blinov, D. L. Moehring, L.-M. Duan, and C. Monroe, Observation of entanglement between a single trapped atom and a single photon, Nature (London) 428, 153 (2004).

[91] J. Volz, M. Weber, D. Schlenk, W. Rosenfeld, J. Vrana, K. Saucke, C. Kurtsiefer, and H. Weinfurter, Observation of Entanglement of a Single Photon with a Trapped Atom, Phys. Rev. Lett. 96, 030404 (2006).

[92] T. Wilk, S. C. Webster, A. Kuhn, and G. Rempe, Singleatom single-photon quantum interface, Science 317, 488 (2007).

[93] E. Togan, Y. Chu, A. S. Trifonov, L. Jiang, J. Maze, L. Childress, M. V. G. Dutt, A. S. Sørensen, P. R. Hemmer, A. S. Zibrov, and M. D. Lukin, Quantum entanglement between an optical photon and a solid-state spin qubit, Nature (London) 466, 730 (2010).

[94] D. L. Moehring, P. Maunz, S. Olmschenk, K. C. Younge, D. N. Matsukevich, L.-M. Duan, and C. Monroe, Entanglement of single-atom quantum bits at a distance, Nature (London) 449, 68 (2007).

[95] D. Cozzolino, B. D. Lio, D. Bacco, and L. K. Oxenløwe, Highdimensional quantum communication: Benefits, progress, and future challenges, Adv. Quantum Technol. 2, 1900038 (2019).

[96] N. Brahms and D. M. Stamper-Kurn, Spin optodynamics ana$\log$ of cavity optomechanics, Phys. Rev. A 82, 041804(R) (2010).

[97] F. Brennecke, T. Donner, S. Ritter, T. Bourdel, M. Köhl, and T. Esslinger, Cavity QED with a Bose-Einstein condensate, Nature (London) 450, 268 (2007).

[98] Y. Colombe, T. Steinmetz, G. Dubois, F. Linke, D. Hunger, and J. Reichel, Strong atom-field coupling for Bose-Einstein condensates in an optical cavity on a chip, Nature (London) 450, 272 (2007).

[99] L. M. Norris, C. M. Trail, P. S. Jessen, and I. H. Deutsch, Enhanced Squeezing of a Collective Spin via Control of Its Qudit Subsystems, Phys. Rev. Lett. 109, 173603 (2012).

[100] G. Vitagliano, I. Apellaniz, I. L. Egusquiza, and G. Tóth, Spin squeezing and entanglement for an arbitrary spin, Phys. Rev. A 89, 032307 (2014).

[101] V. A. Dzuba, V. V. Flambaum, and B. L. Lev, Dynamic polarizabilities and magic wavelengths for dysprosium, Phys. Rev. A 83, 032502 (2011). 